\title{
Multi-method-modeling of interacting galaxies
}

\section{A unique scenario for NGC 4449 ?}

\author{
Ch. Theis ${ }^{1}$ and S. Kohle ${ }^{2,3}$ \\ 1 Institut für Theoretische Physik und Astrophysik der Universität Kiel, Olshausenstr. 40, 24098 Kiel, Germany \\ 2 Radioastronomisches Institut der Universität Bonn, Auf dem Hügel 71, 53121 Bonn, Germany \\ 3 Center for Medical Diagnostic Systems and Visualization MeVis GmbH at University of Bremen, \\ Universitätsallee 29, 28359 Bremen, Germany \\ e-mail: sven@mevis.de
}

Received 9 July 1999 / Accepted 3 January 2001

\begin{abstract}
NGC 4449 is an active star-forming dwarf galaxy of Magellanic type. From radio observations, van Woerden et al. (1975) found an extended HI-halo around NGC 4449 which is at least a factor of 10 larger than the optical diameter $D_{25} \approx 5.6 \mathrm{kpc}$. Recently, Hunter et al. (1998) discerned details in the HI-halo: a disc-like feature around the center of NGC 4449 and a lopsided arm structure. We combined several $N$-body methods in order to investigate the interaction scenario between NGC 4449 and DDO 125, a close companion in projected space. In a first step fast restricted $N$-body models are used to confine a region in parameter space reproducing the main observational features. In a second step a genetic algorithm is applied for a uniqueness test of our preferred parameter set. We show that our genetic algorithm reliably recovers orbital parameters, provided that the data are sufficiently accurate, i.e. all the key features are included. In the third step the results of the restricted $N$-body models are compared with self-consistent $N$-body simulations. In the case of NGC 4449 , the applicability of the simple restricted $N$-body calculations is demonstrated. Additionally, it is shown that the HI gas can be modeled here by a purely stellar dynamical approach. In a series of simulations, we demonstrate that the observed features of the extended HI disc can be explained by a gravitational interaction between NGC 4449 and DDO 125. According to these calculations the closest approach between both galaxies happened $\sim 4-610^{8}$ yr ago at a minimum distance of $\sim 25 \mathrm{kpc}$ on a parabolic or slightly elliptic orbit. In the case of an encounter scenario, the dynamical mass of DDO 125 should not be smaller than $10 \%$ of NGC 4449's mass. Before the encounter, the observed HI gas was arranged in a disc with a radius of $35-40 \mathrm{kpc}$ around the center of NGC 4449 . It had the same orientation as the central ellipsoidal HI structure. The origin of this disc is still unclear, but it might have been caused by a previous interaction.
\end{abstract}

Key words. galaxies: interactions - galaxies: kinematics and dynamics - individual: NGC 4449, DDO 125 methods: $N$-body simulations - methods: data analysis

\section{Introduction}

During the last decades the classical picture of galaxies being Weltinseln (e.g. Kant 1755; von Humboldt 1850), i.e. island universes, has completely changed. In the 1920s, galaxies were thought to be in general islands, i.e. isolated stellar systems, with just a few obvious exceptions like the M 51 system. Systems not fitting the Hubble classification have been neglected - by definition - as peculiar. Interest in these systems has increased strongly since the new catalogues by Vorontsov-Velyaminov (1959) and Arp (1966) became available. They demonstrated that even peculiar objects have common features like bridges, tails,

Send offprint requests to: Ch. Theis,

e-mail: theis@astrophysik.uni-kiel.de or rings. Better observational instruments allowing for deeper images and new wavelength ranges have revealed more complex structures. Galaxies formerly classified as non-interacting may have had some gravitational interaction in the past which is still reflected in e.g. warps, flares, or thick discs. Especially useful are HI observations which can cover a much larger radial extension than the optical images. Therefore, HI is a very good tracer for tidal interactions.

The theoretical understanding of interacting galaxies suffered for a long time from the lack of computational power allowing for a numerical solution of the gravitational $N$-body problem. After a remarkable treatment by Holmberg (1941) who built an analogue computer (consisting of light bulbs and photo cells) to determine the 
gravitational force, it took 20 years until $N$-body simulations were performed on a general purpose computer (Pfleiderer \& Siedentopf 1961): the basic idea of these restricted $N$-body simulations is the assumption that the potential of interacting galaxies can be adequately modeled by two particles which represent the galactic masses and move under their mutual gravitation, i.e. on Keplerian orbits. With these assumptions all the other particles are just test particles, and the complete $N$-body problem is reduced to $N$ single body problems for a time-dependent potential. In a remarkable series of simulations Toomre \& Toomre (1972) applied this technique to determine the parameters of some well-studied interacting systems like Arp 295, M 51 + NGC 5195, or NGC 4038/39.

New $N$-body techniques have been developed which increased the accessible particle number by many orders of magnitude. E.g. the TREE-method (Barnes \& Hut 1986; Hernquist 1987, 1990) in which the organization of the force calculation - the most time-consuming part of $N$ body calculations - adapts to clumpy mass distributions for a moderate computational price $(\sim \mathrm{O}(N \log N))$ compared to direct simulations $\left(\sim \mathrm{O}\left(N^{2}\right)\right)$. By this, Barnes (1988) was able to simulate encounters of disc galaxies including all dynamical components, i.e. the disc, the bulge, and the halo as $N$-body systems. Compared to faster grid-based methods (e.g. Sellwood 1980) or expansion methods (e.g. Hernquist \& Ostriker 1992) direct $N$ body simulations (or semi-direct methods like TREEs) are more flexible with respect to strongly varying geometries and scalelengths. An alternative to these techniques are special-purpose computers (such as the machines of the GRAPE project, Sugimoto et al. 1990). They implemented Newton's law of gravity (modified for gravitational softening) in the hardware which allows for a very fast direct determination of the gravitational forces (however, there still exists the $N^{2}$ bottleneck). E.g. for simulations with $N=10^{5}$ particles a GRAPE3af (with 8 GRAPE processors) is competitive with a TREE-code running on a CRAY T90.

Two main methods (based on particles) to treat a dissipative, star-forming gas have been applied: the smoothed particle hydrodynamics scheme ( $\mathrm{SPH}$ ) solves the gasdynamical equations, by this emphasizing the diffuse nature of the interstellar medium (ISM) (e.g. Hernquist \& Katz 1989). An alternative approach focuses on the clumpiness of the ISM treated as sticky particles: without physical collisions or close encounters of clouds they move in ballistic orbits like stars. However, in the case of a collision the clouds might merge or lose kinetic energy depending on the adopted microphysics (e.g. Casoli \& Combes 1982; Palouš et al. 1993; Theis \& Hensler 1993).

Relatively few papers exist modeling special objects. This deficiency is the result of two factors: first high resolution data in configuration and velocity space are required, covering a large fraction of the space between the interacting galaxies. In principle, HI would be suitable, however, there are just a few observational sites which give data of sufficient quality.
The second problem is the large parameter space for a galactic interaction resulting in two connected difficulties: finding a good fit and determining its uniqueness (or other acceptable parameter sets). Observationally, only three kinematical quantities - the projected position on the sky and the line-of-sight velocity - can be measured. Another parameter, the galactic mass, depends on the availability of velocity data, the determination of the distance, and the reliability of the conversion from velocity to masses. Neglecting the center-of-mass data of the interacting system these 14 parameters reduce to 7 parameters containing the relative positions and velocities. These 7 values just fix the orbit in the case of a two-body interaction. Moreover, one has to specify the parameters that characterize both stellar systems, e.g. characteristic scales, orientation, or rotation. The final result is a highdimensional parameter space which is in general too large for a standard search method. For instance, the interaction of a galactic disc with a point-mass galaxy is described at least by 7 parameters. A regular grid with a poor coverage of 10 grid points per dimension demands $10^{7}$ models or 3400 years GRAPE simulation time (assuming 3 CPUhours for a single simulation) or still about a year with a restricted $N$-body program (assuming 3 CPU-seconds per simulation).

An alternative approach to investigate large parameter spaces is a genetic algorithm (Holland 1975; Goldberg 1989). This applies an evolutionary mechanism on a population (i.e. a set of parameters), from which members are selected for parenthood according to their fitness. Fitness is determined by the ability of the parameters to match the observations, whereas the reproduction within the genetic algorithm is done by cross-over of the parental parameters and applying a mutation operator. Although genetic algorithms (GA) have been used in many branches of science, there are just a few applications in astrophysics, e.g. for fitting rotation curves or analysis of Doppler velocities in $\delta$ Scuti stars (for a review see Charbonneau 1995). Recently, Wahde (1998) demonstrated the ability of genetic algorithms to recover orbital parameters for artificial data generated by $N$-body simulations of strongly interacting galaxies. He stressed that in these systems the positional information can be already sufficient to determine the orbital elements.

In this paper we combine different $N$-body techniques in order to create a model for the dynamics of interacting galaxies. In a first step, restricted $N$-body simulations are performed to constrain the model parameters: if a sufficiently accurate data set is available, the genetic algorithm can be used to find the orbital parameters and to check the uniqueness of the result. Additionally, the sensitivity of the solution can be checked by an extended parameter study. Alternatively, in the case of insufficient data, one can use the restricted $N$-body models to obtain a first guess of the parameters, creating an artificial intensity map and then apply the genetic algorithm to check its uniqueness. In the second step, self-consistent models are necessary in order to tune the parameters and to 
check the applicability of the restricted $N$-body method. These checks must address two questions: is the neglection of self-gravity acceptable? Does gas dynamics alter the results significantly? Technically this means that selfconsistent simulations with and without gas have to be performed.

The numerical models of this paper are performed on NGC 4449 which is a prototype actively star-forming galaxy similar in size and mass to the Large Magellanic Cloud. NGC 4449 is surrounded by an extended HI halo which shows some distortion (van Woerden et al. 1975; Bajaja et al. 1994). Hunter et al. (1998) published high resolution HI images showing a large scale lopsided structure. Though there seems to be a close dwarf companion, DDO 125, it is not clear whether the observed streamers are tidal tails caused by an interaction with DDO 125 (Hunter et al. 1998). For example, tidal distortions are missing in the optical image of both galaxies and no clear bridge has been detected. Theis \& Kohle (1998) demonstrated qualitatively by $N$-body simulations that the main HI features could stem from a face-on encounter provided the mass of DDO 125 exceeds $10 \%$ of the mass of NGC 4449. An alternative scenario assumes that the HI structure is an ongoing infall of gas onto NGC 4449, possibly caused by the encounter with DDO 125 followed by a compression and cooling of the gas (Silk et al. 1987). However, it is puzzling that the observed large-scale, lopsided and regular structure in NGC 4449 can be maintained over timescales such as the orbital period which is longer than $1 \mathrm{Gyr}$ for the outer streamer. The high internal velocity dispersion of $10 \mathrm{~km} \mathrm{~s}^{-1}$ would destroy the streamers on a timescale of $210^{8} \mathrm{yr}$ (Hunter et al. 1998). In this paper we investigate this interaction scenario, addressing the question of whether the observed structures can actually be formed by tidal interaction and which constraints on the mass distribution or the orbits of both galaxies can be derived.

Section 2 summarizes the observational data of NGC 4449 and DDO 125 relevant for the models of this paper. The different modeling techniques and the numerical results are described in Sect. 3 (the restricted $N$-body method is introduced (Sect. 3.1) and a set of first results is shown in Sects. 3.2 and 3.3). The genetic algorithm approach is briefly introduced in Sect. 3.4 and explained in more detail in the Appendix. Its results are shown in Sect. 3.5. Finally, the restricted $N$-body simulations are compared with self-consistent models with (Sect. 3.7) and without gas (Sect. 3.6). The results of these different approaches are summarized and discussed in Sect. 4 .

\section{The case of NGC 4449 and DDO 125}

\subsection{NGC 4449}

When optical images of NGC 4449 and the Large Magellanic Cloud are compared, many similarities like the presence of a bar, the size or the total luminosity are



Fig. 1. Contour map of the integrated HI-intensity of NGC 4449. NGC 4449 is at the center of the image and DDO 125 is located at the almost circular contours in the South. Details are given in Hunter et al. (1998). This image was kindly provided by Deidre Hunter

found. However, unlike the LMC NGC 4449 is a candidate for studies of the properties of a Magellanic-type irregular unaffected by the tidal field of a large galaxy. Its distance of 2.9-5.6 $\mathrm{Mpc}^{1}$ allows for detailed investigations, performed in the visual (e.g. Crillon \& Monnet 1969; Hunter \& Gallagher 1997), in the radio band (e.g. van Woerden et al. 1975; Klein et al. 1996), in the HI emission line (e.g. Bajaja et al. 1994; Hunter \& Gallagher 1997; Hunter et al. 1998), in the CO emission line (e.g. Sasaki et al. 1990; Hunter \& Thronson 1996; Kohle et al. 1998), in the infrared (e.g. Hunter et al. 1986), in the FUV (e.g. Hill et al. 1994) and in X-rays (e.g. della Ceca et al. 1997; Bomans \& Chu 1997). Inside the Holmberg diameter of $11 \mathrm{kpc}$, NGC 4449 shows ongoing strong star formation along a bar-like structure (Hill et al. 1994). This activity seems to induce a variety of morphological features like filaments, arcs, or loops which have a size of several kpc (Hunter \& Gallagher 1997).

Early radio observations of van Woerden et al. (1975) exhibited a large HI structure exceeding the Holmberg diameter of $11 \mathrm{kpc}$ by a factor of 7.5. Recent observations by Bajaja et al. (1994) with the Effelsberg telescope and by Hunter et al. (1998) with the VLA revealed a complex structure of several components (Fig. 1): an elongated

\footnotetext{
${ }^{1}$ Most of the distance estimates for NGC 4449 differ because of the assumed value $H_{0}$ for the Hubble constant. Independently of $H_{0}$, Karachentsev \& Drozdovsky (1998) derived from the photometry of the brightest blue stars a distance of $2.9 \mathrm{Mpc}$. However, the error of this measurement is unclear. In accordance with Hunter et al. (1998) a distance of $3.9 \mathrm{Mpc}$ will be assumed in this paper. Thus, $1^{\prime}$ corresponds to $1.1 \mathrm{kpc}$.
} 
ellipse of HI gas centered on NGC 4449 has a total mass of $\sim 1.110^{9} M_{\odot}$. This gas rotates rigidly inside $11 \mathrm{kpc}$, reaching a level of $97 \mathrm{~km} \mathrm{~s}^{-1}$ in the deprojected velocity. Outside $11 \mathrm{kpc}$, the rotation curve is flat. Bajaja et al. (1994) derived a dynamical mass of $7.010^{10} M_{\odot}$ inside $32 \mathrm{kpc}$ and a HI gas mass fraction of $3.3 \%$. Within $11 \mathrm{kpc}$ the dynamical mass is $2.310^{10} M_{\odot}$ and the mass inside the optical radius of about $5.6 \mathrm{kpc}$ is $310^{9} M_{\odot}$. Applying single dish data for a $21^{\prime}$ beamwidth, Hunter et al. (1998) derived about $10^{10} M_{\odot}$. The HI gas inside the optical part of NGC 4449 counterrotates with respect to the outer regions. For the central ellipse, Hunter et al. (1998) determined a position angle of $230^{\circ} \pm 17^{\circ}$ and an inclination of $60^{\circ} \pm 5^{\circ}$.

South of the galactic center, a streamlike structure of $25 \mathrm{kpc}$ emanates which abruptly splits into two parts: a small spur (5 kpc) which points towards DDO 125, a close irregular galaxy, and a long extended stream. The latter first points $24 \mathrm{kpc}$ to the north (in the following: vertical streamer), then $49 \mathrm{kpc}$ north-east and, finally, $27 \mathrm{kpc}$ to the south-east (together called the northeastern streamer), covering $180^{\circ}$ around NGC 4449. The long straight sections are notable, as are the rather abrupt changes of direction. The total mass of the streamers measured by the VLA observations is about $110^{9} M_{\odot}$. Hunter et al. (1998) point out that compared to the Effelsberg data there is an additional diffuse HI mass not detected in the VLA observations which amounts to two-thirds of the HI in the extended structures.

\section{2. $D D O 125$}

DDO 125 is located in the south-east of NGC 4449 at a projected distance of $41 \mathrm{kpc}$. The line-of-sight velocity difference between DDO 125 and NGC 4449 is only about $10 \mathrm{~km} \mathrm{~s}^{-1}$. Tully et al. (1978) found rigid rotation inside the optical radius of DDO 125 and an inclination of the adopted disc of $50^{\circ}$. From this they derived a total mass of $510^{8} M_{\odot}$ (corrected for a distance of $3.9 \mathrm{Mpc}$ ) which is in agreement with single dish observations (21' beamwidth) of Fisher \& Tully (1981). However, Ebneter et al. (1987) reported VLA-observations which show a linear increase of the rotation curve out to the largest radii where HI has been detected, i.e. out to twice the optical radius. This already allows for an estimate of a lower mass limit for DDO 125 which is a factor 8 larger than the value of Tully et al. (1978), i.e. $410^{9} M_{\odot}$. Since neither flattening of the rotation curve nor any decline has been observed, this value is only a lower limit. If one compares that mass with the total mass of NGC 4449 derived by Bajaja et al. (1994) one gets a mass ratio of about $q \approx 5.7 \%$ which is, however, just a lower limit. Comparison of the masses inside the range of rigid rotation gives a higher mass ratio of $q \approx 18 \%$.

\section{Numerical modeling}

For the simulations described in this paper three different $N$-body approaches have been applied: the method of restricted $N$-body simulations (Pfleiderer \& Siedentopf 1961; Toomre \& Toomre 1972) is described in Sect. 3.1. The self-gravitating models are split into calculations without gas (Sect. 3.6) and simulations which include gas by means of sticky particles (Sect. 3.7).

The advantage of the first method is twofold: the reduction of the full $N$-body problem to $N$ single body problems and the use of particles as test particles which gives high spatial resolution at the places of interest for almost no computational cost. As a direct consequence, restricted $N$-body calculations can be performed with fewer particles than self-consistent simulations which strongly suffer from two-body relaxation in case of small particle numbers. Furthermore, the CPU-time scales linearily with particle number which is superior to almost all $N$-body codes. E.g. a restricted $N$-body simulation sufficiently reproduces tidal features with only 1000 particles, whereas a direct self-consistent calculation needs an (optimistic) minimum of 10000 particles to reproduce a stable galactic disc on the interaction timescale. Comparing the CPUtimes of such a self-consistent simulation performed on a GRAPE3 board with the values of a restricted $N$-body simulation we get a time saving of at least a factor of $1000^{2}$. Hence, the restricted $N$-body calculation is at the moment the only method which allows about $10^{4}$ simulations in a couple of CPU-hours. Such a fast computation is a prerequisite for all systematic searches in parameter space such as the genetic algorithm described in Sect. 3.4.

However, the simplified treatment of the galactic potential in restricted $N$-body simulations prohibits any feedback of the tidal interaction on the orbits of both galaxies, e.g. no transfer of orbital angular momentum into galactic spin is possible (i.e. no merging). To overcome this problem, the results of restricted $N$-body models and genetic algorithms must be compared with detailed selfconsistent simulations. In a first step the stellar dynamical applicability of the restricted models is checked by a comparison with self-gravitating systems calculated with a GRAPE3af special purpose computer (Sugimoto et al. 1990; see Sect. 3.6).

So far the dynamics of the system have been investigated in terms of stellar dynamics, though in many cases (such as the case of NGC 4449) HI gas is observed. In general, the effects of neglecting gas dynamics are not clear. E.g. a purely stellar dynamical ansatz was successfully applied for the system M 81 and NGC 3077 (Thomasson \& Donner 1993), whereas in other systems the dynamics of the gas can deviate strongly from the behaviour of the stars (e.g. Noguchi 1988; Barnes \& Hernquist 1996). Therefore, one has to compare stellar and gas dynamical results which is done in this paper by a sticky particle method (Sect. 3.7).

\footnotetext{
2 The exact acceleration depends strongly on the details of the comparison, e.g. the requirements on disc stability and therefore the number of particles for the self-consistent model, the detailed orbits, the method of the self-consistent calculation etc. An acceleration of a factor 1000 is only a lower limit.
} 
The units are chosen as $1 \mathrm{kpc}, 710^{10} M_{\odot}$ (the dynamical mass of NGC 4449) and $G=1$. This results in a time unit of $1.78 \mathrm{Myr}$ and a velocity of $549 \mathrm{~km} \mathrm{~s}^{-1}$.

\subsection{Restricted N-body models}

Restricted $N$-body simulations aim to reduce the $N$-body problem to $N$ 1-body problems by assuming that the gravitational potential is given by a simple relation. E.g. twoor a few-body problems have known (semi-)analytical solutions or can be solved by fast standard methods. Here we assume that the gravitational forces on each particle are given by the superposition of the forces exerted by two point-like objects (the galaxies) moving on Keplerian orbits. Thus, for the orbits one has to specify 14 parameters which reduce to 7 in the center-of-mass frame (relative coordinates/velocities and the mass ratio): the orbital plane is fixed by the inclination angle and the argument of the ascending node. The orbit itself is characterized by its eccentricity and the location of the pericenter, i.e. the pericentric distance and the argument of the pericenter. Finally, mass and initial location of the reduced particle (or the time of pericentric passage) have to be specified to fix the phase. For a comparison with the observations, the phase of the observation also must be supplied. Three of the seven orbital parameters can be fixed by the observed location of both galaxies on the plane of sky ( $x-y$ plane) and the line-of-sight velocity. The masses (and thus the mass ratio) can be estimated by the velocity profiles of both galaxies, provided the distance to them is known.

In this paper, the time of pericentric passage is set to zero. As input, the orbital eccentricity and inclination and the minimum distance are given. Furthermore, the directly observable parameters, i.e. the masses of the galaxies, the projection of the relative positions of both galaxies onto the plane of sky, and their line-of-sight velocity difference, are supplied. They are used to determine the argument of the pericenter, the phase (or time since pericentric passage) of the observed system and the argument of the ascending node of the orbital plane. Additionally, the initial distance or phase has to be specified: in the case of parabolic and hyperbolic encounters, the distance was set to $100 \mathrm{kpc}$, which guarantees a sufficiently low tidal influence at the start of the simulation. In the case of an elliptical encounter, the situation is more difficult, because the apocentric distance might be small, especially if the eccentricity is low. Thus, the system might be tidally affected all the time or by a series of repeated encounters which makes the applicability of the restricted $N$-body method doubtful. For these elliptical orbits, the initial azimuthal angle of the orbit was set to $120^{\circ}\left(0^{\circ}\right.$ corresponds to pericenter).

The test particles are arranged in a flat disc moving on circular orbits. The disc itself is characterized by its orientation, i.e. its inclination and position angle, the scalelength and the outer edge. Additionally, an inner edge can be specified in order to avoid a waste of computational time by integrating tidally unaffected orbits well inside the tidal radius of the galaxy. In most of the restricted $N$-body simulations the test particles are distributed in 30 to 60 equidistant rings of 125 particles, i.e. a total of $N=3750$ to 7500 particles. The typical spacing of the rings is 0.7 to $1.5 \mathrm{kpc}$ depending on the outer edge of the disc. The time integration of the equations of motion is performed by a Cash-Karp Runge-Kutta integrator of fifth order with adaptive timestep control (Press et al. 1992). In order to prevent a vanishingly small timestep by an accidental infall of a test particle to the galactic center, the gravitational force is softened on a length scale of $1 \mathrm{kpc}$. In order to speed-up the calculation the galactic positions are tabulated by cubic splines and the integrator uses these look-up tables. On a SPARC10 with a $150 \mathrm{MHz}$ CPU a typical 7500-particle encounter takes 23 CPU seconds, which can be reduced by a factor of $2-3$ by applying an inner edge of $10 \mathrm{kpc}$.

\subsection{A first guess}

In a series of simulations the orbital parameters (minimum distance $d_{\min }$, the eccentricity $\epsilon$, the mass ratio $q$ and the orientation of the orbital plane) as well as the disc parameters (size $R_{\max }$ of the disc, its position angle $\alpha$ and inclination $i$ ) are varied (see Table 1).

The evolution of the primary galaxy (NGC 4449) in the reference model A is displayed in Fig. 2. At the beginning, both galaxies have a distance of $100 \mathrm{kpc}$ corresponding to $995 \mathrm{Myr}$ before closest approach. The particles inside $10 \mathrm{kpc}$ are omitted for an easier identification of the evolving structure. This does not alter the results, since the related particles are only weakly affected by the interaction, as the persistence of the elliptical shape of the central hole demonstrates. At the moment of closest approach $(t=0)$, only the outer region of NGC 4449's disc reacts on the intruder. $71 \mathrm{Myr}$ later (i.e. system time 40), a dense outer rim, starting at the projected position of DDO 125 and pointing to the north, begins to form. Secondly, a weak bridge-like feature seems to connect both galaxies. Both features are more pronounced after $178 \mathrm{Myr}$. At that time an additional tidal feature begins to evolve north of the center. When DDO 125 has a projected distance of $41 \mathrm{kpc}$ (362 Myr after closest approach), three features are discernible: the remnant of the former bridge starts south of the center of NGC 4449 pointing to the south-west. The vertical feature starts at the end of the first structure and points to the north. The third structure is a long tidal arm which starts west of NGC 4449 pointing to north-east before turning back to the south-east.

A comparison of the intensity map of model A (lower right diagram in Fig. 2) with the observations (Fig. 1) demonstrates that the main observational features can be reproduced qualitatively by the reference model A, i.e. a parabolic orbit, a mass ratio $q=0.2$ and a minimum distance $d_{\min }=25 \mathrm{kpc}$. The parameters for the disc orientation are chosen in agreement with Hunter et al.'s 

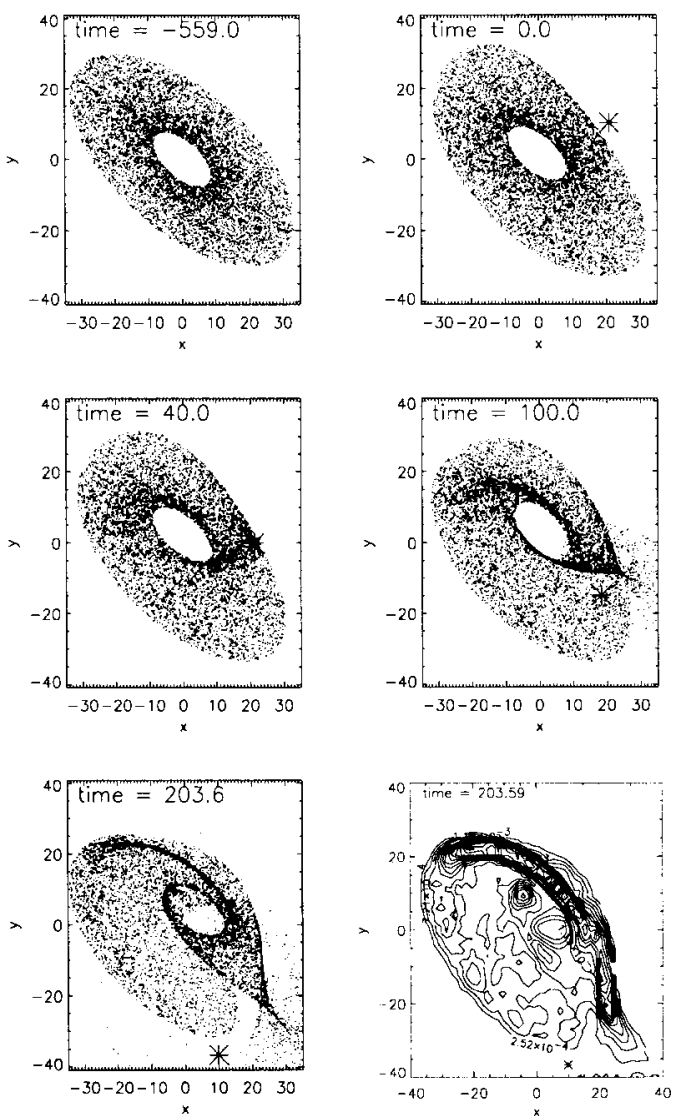

Fig. 2. Temporal evolution of the particle positions around NGC 4449 projected onto the plane-of-sky for different times. The perturber DDO 125 is shown by the star. Closest approach is at $t=0$. The final diagram corresponds to a projected distance of $41 \mathrm{kpc}$. The mass ratio of both galaxies is set to $q=0.2$. The original $\mathrm{HI}$ disc has an inclination of $i=60^{\circ}$, a position angle $\alpha=230^{\circ}$ and a radial extent of $R_{\max }=40 \mathrm{kpc}$. The particles inside a radius of $10 \mathrm{kpc}$ are omitted. The contour lines in the lower right diagram show the normalized surface density distribution corresponding to the particle distribution in the lower left diagram. The intensities are calculated on a $50 \times 50$ grid and smoothed over 3 grid cells in each dimension. One time unit corresponds to $1.78 \mathrm{Myr}$

suggestion of $i=60^{\circ}, \alpha=230^{\circ}$, whereas the disc radius was set to $R_{\max }=40 \mathrm{kpc}$. Although this numerical model is not a detailed fit of the data, the characteristic sizes and locations of the streamers as well as the unaffected disc can be found. The material located between the streamer and the disc, which is not seen in Hunter's data, corresponds to the extended HI mass detected in the single dish observations by van Woerden et al. (1975). In the following sections we will discuss the influence of the individual parameters. The global uniqueness of the reference model is investigated in Sect. 3.5.

\subsection{Parameter study}

In this section the influence of the basic parameters, i.e. the orientation of the orbital plane, the orbital
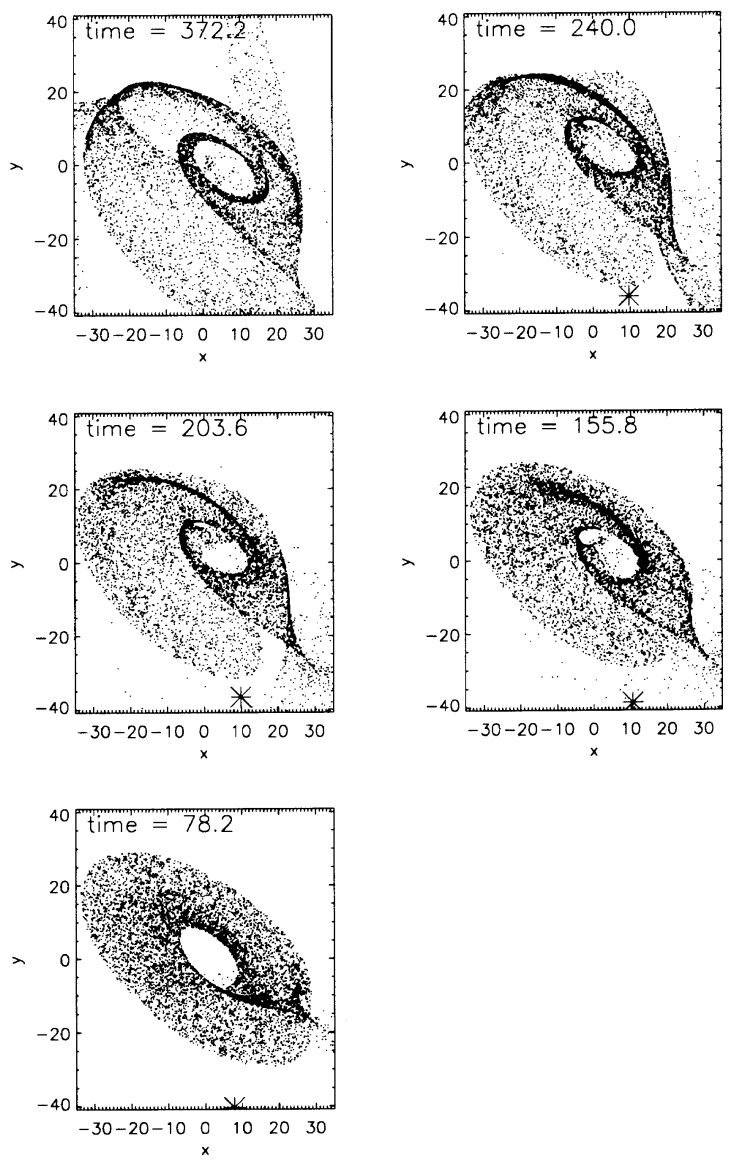

Fig. 3. Dependence on the eccentricity. Projection of the particle positions onto the plane-of-sky at the moment of a projected distance of $41 \mathrm{kpc}$. The eccentricities of the models are 0.5 (upper left, model B1), 0.8 (upper right, B2), 1.0 (A, reference model), 1.5 (B3) and 5.0 (B4)

eccentricity and the minimum distance, the mass ratio of both galaxies, and the orientation of the HI disc and its extent are discussed (see Table 1). In the following simulations, all parameters except the one of interest are kept constant with respect to model A. Thus, the vicinity of the reference model in parameter space is studied.

Eccentricity. The eccentricity mainly influences the interaction timescale which is defined here as the time between maximum approach and a projected distance of $41 \mathrm{kpc}$, i.e. the moment of observation. An eccentricity of 5.0 (corresponding to a hyperbolic encounter) decreases the interaction time by a factor 2.6 resulting in less structural changes of the primary galaxy (Fig. 3): only the remnant of the bridge feature can be discerned, whereas the other two streamers found in the reference model are missing completely. With decreasing eccentricity, the northeastern streamer becomes pronounced before the vertical structure arises. The latter is weakly expressed for $\epsilon=1.5$. Reducing $\epsilon$ further to bound orbits makes the features even more prominent. In the case of $\epsilon=0.5$ the northeastern and the vertical streamer form a single structure clearly detached from the center. However, due to the 
Table 1. Parameters of the different restricted $N$-body models: model name (Col. 1), orbital eccentricity $\epsilon$ (Col. 2), mass ratio $q$ of both galaxies (Col. 3), minimum separation $d_{\text {min }}$ of galactic centers (in kpc, Col. 4), inclination $i$ of orbital plane (in degrees, Col. 5), orientation of NGC 4449 disc (in degrees): inclination $i_{\mathrm{d}}$ (Col. 6) and position angle $\alpha_{\mathrm{d}}$ (Col. 7), maximum radius $R_{\mathrm{max}}$ of gaseous disc (in kpc, Col. 8)

Model parameters of the restricted $N$-body simulations

\begin{tabular}{|l||c|c|c|c||c|c|c||l|}
\hline model & $\epsilon$ & $q$ & $d_{\text {min }}$ & $i$ & $i_{\mathrm{d}}$ & $\alpha_{\mathrm{d}}$ & $R_{\max }$ & comment \\
\hline \hline A & 1.0 & 0.2 & 25 & 40 & 60 & 230 & 40 & reference model \\
\hline \hline B1 & 0.5 & 0.2 & 25 & 40 & 60 & 230 & 40 & eccentricity \\
\hline B2 & 0.8 & 0.2 & 25 & 40 & 60 & 230 & 40 & \\
\hline B3 & 1.5 & 0.2 & 25 & 40 & 60 & 230 & 40 & \\
\hline B4 & 5.0 & 0.2 & 25 & 40 & 60 & 230 & 40 & \\
\hline \hline C1 & 1.0 & 0.01 & 25 & 40 & 60 & 230 & 40 & mass ratio \\
\hline C2 & 1.0 & 0.05 & 25 & 40 & 60 & 230 & 40 & \\
\hline C3 & 1.0 & 0.1 & 25 & 40 & 60 & 230 & 40 & \\
\hline C4 & 1.0 & 0.3 & 25 & 40 & 60 & 230 & 40 & \\
\hline C5 & 1.0 & 0.4 & 25 & 40 & 60 & 230 & 40 & \\
\hline \hline D1 & 1.0 & 0.2 & 15 & 40 & 60 & 230 & 40 & minimum distance \\
\hline D2 & 1.0 & 0.2 & 20 & 40 & 60 & 230 & 40 & \\
\hline D3 & 1.0 & 0.2 & 30 & 40 & 60 & 230 & 40 & \\
\hline D4 & 1.0 & 0.2 & 35 & 40 & 60 & 230 & 40 & \\
\hline D5 & 1.0 & 0.2 & 40 & 40 & 60 & 230 & 40 & \\
\hline \hline E1 & 1.0 & 0.2 & 25 & 10 & 60 & 230 & 40 & orbital inclination \\
\hline E2 & 1.0 & 0.2 & 25 & 20 & 60 & 230 & 40 & \\
\hline E3 & 1.0 & 0.2 & 25 & 30 & 60 & 230 & 40 & \\
\hline E4 & 1.0 & 0.2 & 25 & 50 & 60 & 230 & 40 & \\
\hline E5 & 1.0 & 0.2 & 25 & 70 & 60 & 230 & 40 & \\
\hline \hline F1 & 1.0 & 0.2 & 25 & 40 & 40 & 230 & 40 & disc inclination \\
\hline F2 & 1.0 & 0.2 & 25 & 40 & 50 & 230 & 40 & \\
\hline F3 & 1.0 & 0.2 & 25 & 40 & 70 & 230 & 40 & \\
\hline \hline G1 & 1.0 & 0.2 & 25 & 40 & 60 & 210 & 40 & pos. angle of disc \\
\hline G2 & 1.0 & 0.2 & 25 & 40 & 60 & 250 & 40 & \\
\hline \hline H1 & 1.0 & 0.2 & 25 & 40 & 60 & 230 & 25 & outer edge of disc \\
\hline H2 & 1.0 & 0.2 & 25 & 40 & 60 & 230 & 30 & \\
\hline H3 & 1.0 & 0.2 & 25 & 40 & 60 & 230 & 35 & \\
\hline H4 & 1.0 & 0.2 & 25 & 40 & 60 & 230 & 50 & \\
\hline
\end{tabular}

strength of the interaction, a second broad tidal arm is ejected from NGC 4449, leaving a low-density region south to the northern tip of the north-eastern streamer.

In order to compare the $\epsilon=0.5$ model with observations, we reran model B1, but with $N=80000$, in order to derive intensity and velocity maps. This model reproduces several observed features very well (Fig. 4): first, the intensity map shows the three observed abrupt changes: at the lower tip of the vertical streamer, at the transition from the vertical streamer to the north-eastern streamer and at the northern tip of the north-eastern streamer. Second, the spatial extent of the streamers is in agreement with the observations. Third, we find a bridge between the northeastern streamer and the central tidally-unaffected part of NGC 4449. Fourth, during the encounter, material is ejected south to NGC 4449. The latter two features can be found in deeper images of NGC 4449 (cf. Fig. 1 of Hunter et al. 1998). However, there are also some remarkable differences: first, both model and observation show a spur at the lower tip of the vertical streamer. However, their directions differ by about $90^{\circ}$. Second, the position of the northern tip of the north-eastern streamer (or the angle between vertical and the north-eastern streamer) differ.

Looking at the velocity map, we find Keplerian rotation in the central region of the simulations, whereas the observations show rigid rotation. The velocities in the streamers reach about $80-100 \mathrm{~km} \mathrm{~s}^{-1}$, which is in agreement with the observed values. Additionally, the velocity field in the simulations shows a small, but significant V-shape, south of NGC 4449 which is 

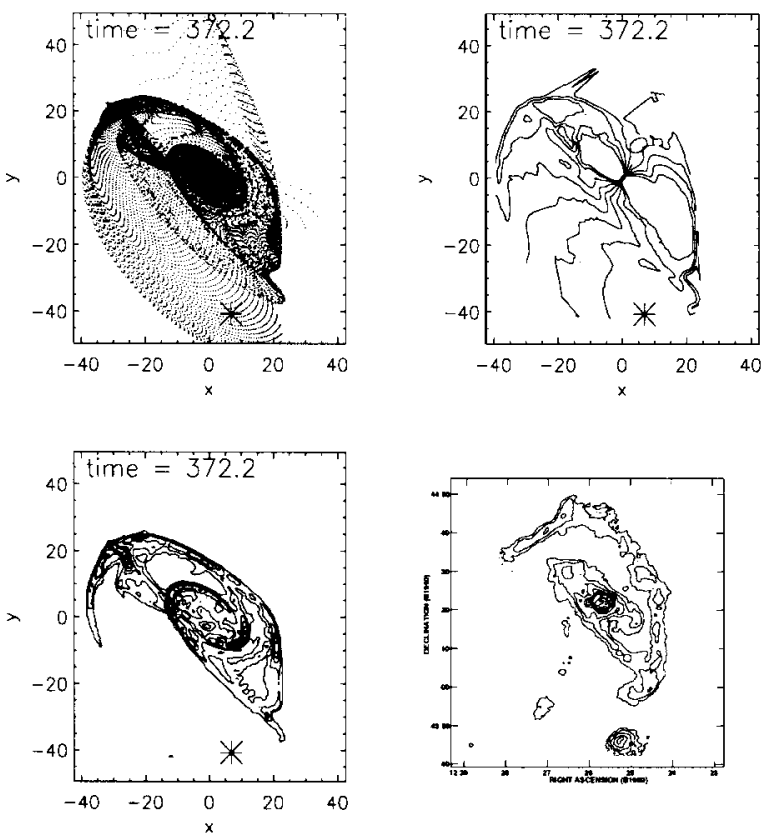

Fig. 4. Projected particle positions (upper left), velocity map (upper right) and intensity map (lower left) for model B1 $(\epsilon=0.5)$ at the moment of a projected distance of $41 \mathrm{kpc}$. The velocity contours differ by $20 \mathrm{~km} \mathrm{~s}^{-1}$, whereas the intensity map ranges from $1 \%$ of the maximum intensity $I_{\max }$ to $I_{\max }$. The star marks the position of DDO 125. The observed intensity map (cf. Fig. 1) is displayed in the lower right panel for comparison

also observed (cf. Fig. 3 of Hunter et al. 1998). The main discrepancies between the observed and numerical velocity maps comes from the point mass approximation of the galactic potential used for the numerical model. Although this approximation must fail for the central region, it should be sufficient for the outer regions which are most affected by tides and which contain almost the entire dynamical mass of NGC 4449.

Mass ratio. The variation of the mass ratio gives a lower limit of about $10 \%$ in order to create a sufficient tidal response (Fig. 5). If the mass ratio exceeds 0.3 , the vertical feature becomes less vertical and the north-eastern streamer is more diffuse, both due to the enhanced tidal pull of the intruder.

Minimum distance. Although the interaction time is not strongly influenced by the minimum distance $d_{\min }$, it determines the maximum strength of the tidal field (Fig. 6): for small separations, the structures become more diffuse and the primary is much more affected. The vertical feature is more extended, creating an additional tidal arm (similar to the model with an orbital eccentricity $\epsilon=0.5$ ). If the distance of closest approach exceeds $30 \mathrm{kpc}$, almost no streamers are formed.

Orbital plane. The orientation of the orbital plane characterizes the observer's location or the line-of-sight,
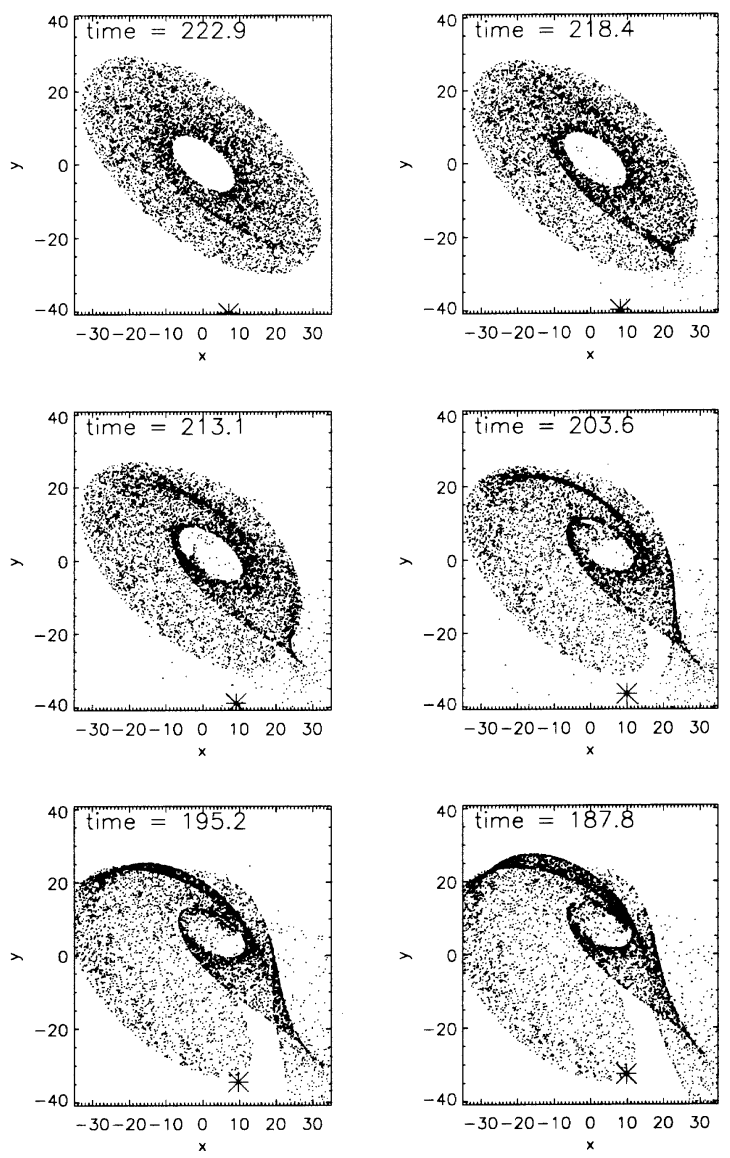

Fig. 5. Dependence on the mass ratio. Projection of the particle positions onto the plane-of-sky at the moment of a projected distance of $41 \mathrm{kpc}$. The mass ratios are - starting with the upper left diagram - 0.01 (model C1), 0.05 (C2), 0.1 (C3), $0.2(\mathrm{~A}$, reference model), $0.3(\mathrm{C} 4)$ and $0.4(\mathrm{C} 5)$

which is fixed by two parameters. For the models here only the orbital inclination is a free parameter, since the second parameter, the position angle of the orbital plane, is fixed by the observed relative line-of-sight velocity of both galaxies. The models show that the inclination affects mainly the orientation of the vertical streamer (Fig. 7): an inclination outside the range $\left[30^{\circ}, 50^{\circ}\right]$ gives too much deviation from the south-north direction of the vertical feature.

HI distribution in NGC 4449. A specification of the mass- and especially the HI distribution is a more difficult task, because, contrary to the well-known two-body problem, the number of free parameters characterizing the structure and internal dynamics of the interacting galaxies is unknown and a matter of investigation itself. For example, it is not a priori clear how the HI is distributed. To reduce the number of free parameters, we will assume here that the observed inner elongated ellipse is the remnant of an original exponential HI disc, which can be specified by its orientation, its scale length and radial extent. The inclination of the disc can roughly be determined from the HI map, whereas its sign is not unambiguous. Placing 



Fig. 6. Dependence on the minimum distance during the encounter. Projection of the particle positions onto the planeof-sky at the moment of a projected distance of $41 \mathrm{kpc}$. The minimum distances are - starting with the upper left diagram - $15 \mathrm{kpc}$ (model D1), $20 \mathrm{kpc}$ (D2), $25 \mathrm{kpc}$ (A, reference model), $30 \mathrm{kpc}$ (D3), $35 \mathrm{kpc}$ (D4) and $40 \mathrm{kpc}$ (D5)

the south-eastern side of the HI disc into the foreground means that the orbital plane of the companion and the plane of the HI disc are nearly orthogonal. We performed several simulations with geometries of this kind, but these show almost no strong tidal responses and they all result in situations completely different to that observed (Kohle 1999). However, placing the north-western side of the HI disc into the foreground and considering the HI velocity field, we now have the companion on a prograde orbit.

Since the particles are treated as test particles, a variation of the scale length does not change the final location of a particle, but rather its mass, which is required for the calculation of the intensity map. A small scale length (e.g. $5 \mathrm{kpc}$ ) would confine too much $\mathrm{HI}$ in the center which is unaffected by the encounter. Since the observations show a large fraction of HI outside $20 \mathrm{kpc}$, we have chosen a large scale length of $30 \mathrm{kpc}$ which gives only a weak radial decline of the HI surface density and, by this, a sufficient amount of $\mathrm{HI}$ in the outer regions.

DiSC SIZE. The influence of the maximum radius $R_{\max }$ of the disc is shown in Fig. 8. If $R_{\max }$ is smaller than $35 \mathrm{kpc}$, the vertical streamer has not been formed at all, indicating that this feature stems from the HI gas far outside the
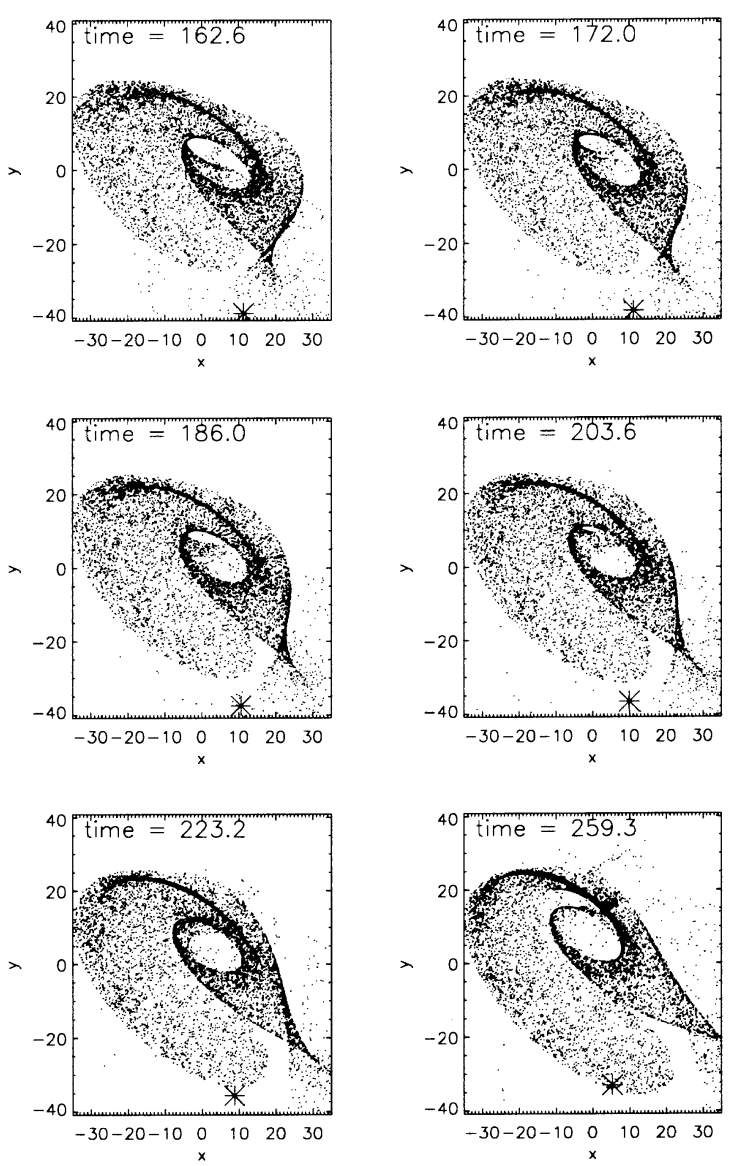

Fig. 7. Dependence on the inclination angle of the orbital plane. Projection of the particle positions onto the plane-ofsky at the moment of a projected distance of $41 \mathrm{kpc}$. The inclinations are $10^{\circ}$ (upper left, model E1), $20^{\circ}$ (upper right, $\mathrm{E} 2), 30^{\circ}(\mathrm{E} 3), 40^{\circ}$ (A, reference model), $50^{\circ}$ (E4) and $70^{\circ}(\mathrm{E} 5)$

center. The north-eastern arm and the bridge, however, are already built up for $R_{\max }=25 \mathrm{kpc}$. With increasing disc size the vertical streamer becomes more pronounced and more extended, which excludes disc sizes larger than $45 \mathrm{kpc}$.

Disc orientation. The orientation of the HI disc also strongly affects the appearance of the primary galaxy. Variation of the position angle $\alpha$ by $20^{\circ}$ gives HI distributions which are incompatible with the observations (Fig. 9). If $\alpha$ is decreased, the vertical streamer is more extended and diffuse. On the other hand, if $\alpha$ is increased, the north-eastern arm becomes too long and the vertical feature vanishes almost completely. Similarly, the results depend strongly on the inclination $i$ : inclinations below $50^{\circ}$ give only a weak bridge and a weak vertical streamer, whereas the orientation of the vertical streamer deviates strongly from the northern direction for inclinations exceeding $70^{\circ}$.

The performed parameter study demonstrates that even close to the parameters of model $\mathrm{A}$, the final particle distribution shows significant variations which are not in agreement with the observations. Thus, the reference model might be a good and locally unique 

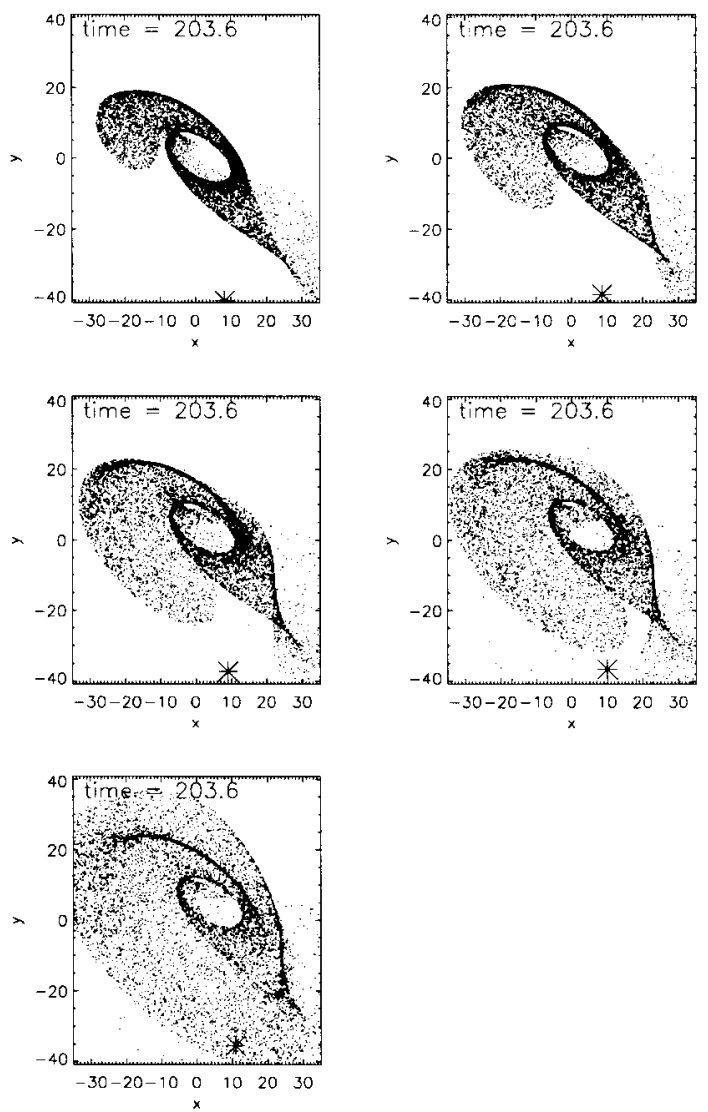

Fig. 8. Dependence on the extension of the HI distribution in NGC 4449. Projection of the particle positions onto the planeof-sky at the moment of a projected distance of $41 \mathrm{kpc}$. The radial extensions are $25 \mathrm{kpc}$ (upper left, model $\mathrm{H} 1$ ), $30 \mathrm{kpc}$ (upper right, H2), $35 \mathrm{kpc}$ (H3), $40 \mathrm{kpc}$ (A, reference model) and $50 \mathrm{kpc}(\mathrm{H} 4)$

candidate describing the dynamics of NGC 4449 and DDO 125. The confidence in this encounter scenario is especially enhanced by the strong dependence on the orientation of the HI disc of NGC 4449. Qualitatively good models are only found if the disc orientation agrees well with the values determined independently by observations. On the other hand, the former parameter study only investigates a small fraction of parameter space and, thus, the uniqueness of the solution is not confirmed. Moreover, in general, it is very tedious to find a good model by hand, i.e. by an unsystematic non-automated search in parameter space. Thus, the probability of accepting the first solution found seems to be very high (especially, if CPUintensive simulations are performed), by this neglecting other regions in parameter space which might also give sufficient fits. In the next section an automatic search in parameter space by means of a genetic algorithm is introduced. This allows in principle for an ab initio determination of acceptable parameter sets (if data sets of sufficient quality are available) or at least for a uniqueness test of a favoured scenario like the reference model described above.
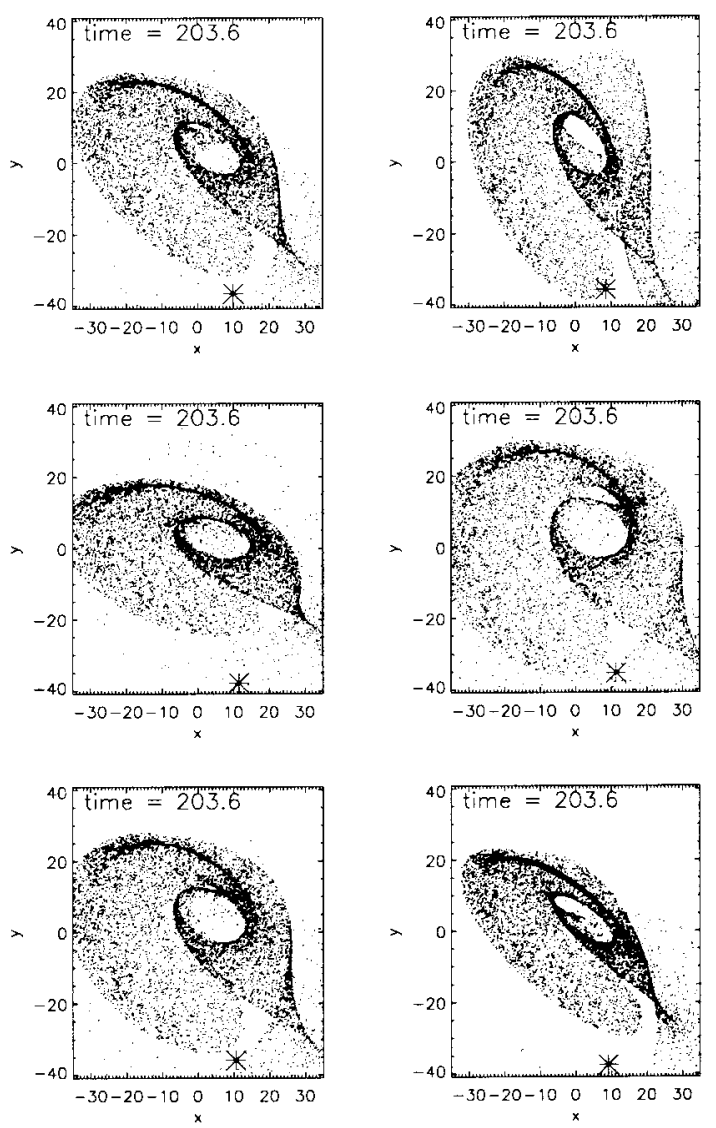

Fig. 9. Dependence on the orientation of the HI disc in NGC 4449. Projection of the particle positions onto the planeof-sky at the moment of a projected distance of $41 \mathrm{kpc}$. The orientation is characterized by the inclination $i_{\mathrm{d}}$ of the disc and the position angle $\alpha_{\mathrm{d}}: i_{\mathrm{d}}=60^{\circ}, \alpha_{\mathrm{d}}=230^{\circ}$ (upper left, model A, reference model); $60^{\circ}, 210^{\circ}$ (upper right, G1); $60^{\circ}$, $250^{\circ}(\mathrm{G} 2) ; 40^{\circ}, 230^{\circ}(\mathrm{F} 1) ; 50^{\circ}, 230^{\circ}(\mathrm{F} 2)$ and $70^{\circ}, 230^{\circ}(\mathrm{F} 3)$

\subsection{The genetic algorithm}

The idea of applying models of organic evolution for optimization problems dates back to the 1960s and 1970s (e.g. Rechenberg 1965; Schwefel 1977). Unlike standard deterministic gradient techniques for optimization (e.g. the downhill simplex method, Press et al. 1992) Rechenberg's Evolutionsstrategie is probabilistic: starting with a more or less random parent, i.e. a single point in parameter space, a child is generated by a random mutation of the parameter set characterizing the parent. The quality of both individuals with respect to the optimization problem (i.e. their fitness ) determines the parent of the next generation. Repeating this process of mutation and selection improves the quality of the individual monotonously. This special implementation of evolutionary algorithms (EAs) in general, has certain advantages: compared to complete grids in parameter space, the probabilistic but oriented nature of the evolutionary search strategy allows for an efficient check of a high-dimensional parameter space. Compared to gradient methods which are very fast near the optimum, EAs do not need any gradient information which might be 

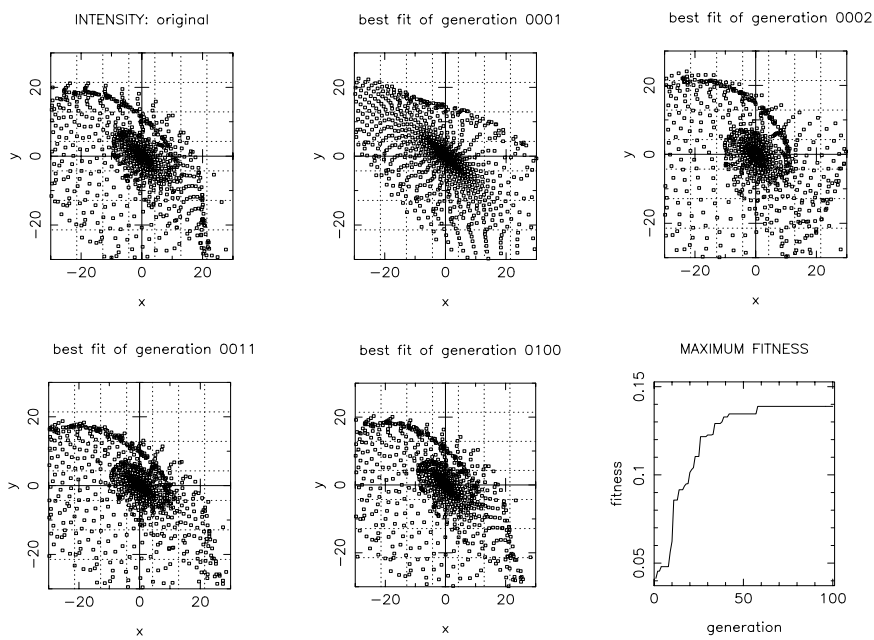

Fig. 10. Best fit model during the course of a GA fitting procedure. The projection of the particles in $x-y$-plane and the corresponding grid for the intensity evaluation are displayed: the original data (upper left), the best fit of the GA after initialization (upper middle), after the first breeding (upper right), after 11 generations (lower left) and at the end of the fitting procedure after 100 generations (lower middle). The evolution of the maximum fitness is shown in the lower right diagram. The number of test particles per simulation is 900

computationally expensive. They depend only weakly on the starting point and - most important - they are able to leave local optima. The price for these features is a large number of fitness evaluations or test points in parameter space before converging to a good solution.

Holland (1975) extended the Evolutionsstrategie of Rechenberg by abandoning asexual reproduction: he used a population of individuals instead of a single individual (and its offspring). From the population two individuals were selected according to their fitness to become parents. These parents represent two points (chromosomes) in parameter space and the corresponding coordinates are treated like genes on a chromosome. These chromosomes are subject to a cross-over and a mutation operation resulting in a new individual which is a member of the next generation. Such a breeding is repeated until the next generation has been formed. Finally, the whole process of sexual reproduction is repeated iteratively until the individuals representing a set of points in parameter space confine sufficiently one or several regions of high fitness. Despite many differences in the detailed implementations of genetic algorithms (GAs), the basic concept of an iterated application of randomized processes like recombination, mutation, and selection on a population of individuals remains in all GAs (Goldberg 1989; Bäck 1996).

\subsection{Are the results unique?}

In order to check the uniqueness of the reference model $\mathrm{A}$, several runs with different parameter sets encoded in genes were performed. Figure 10 shows a typical run of the GA operating on a population of 100 members. The reference
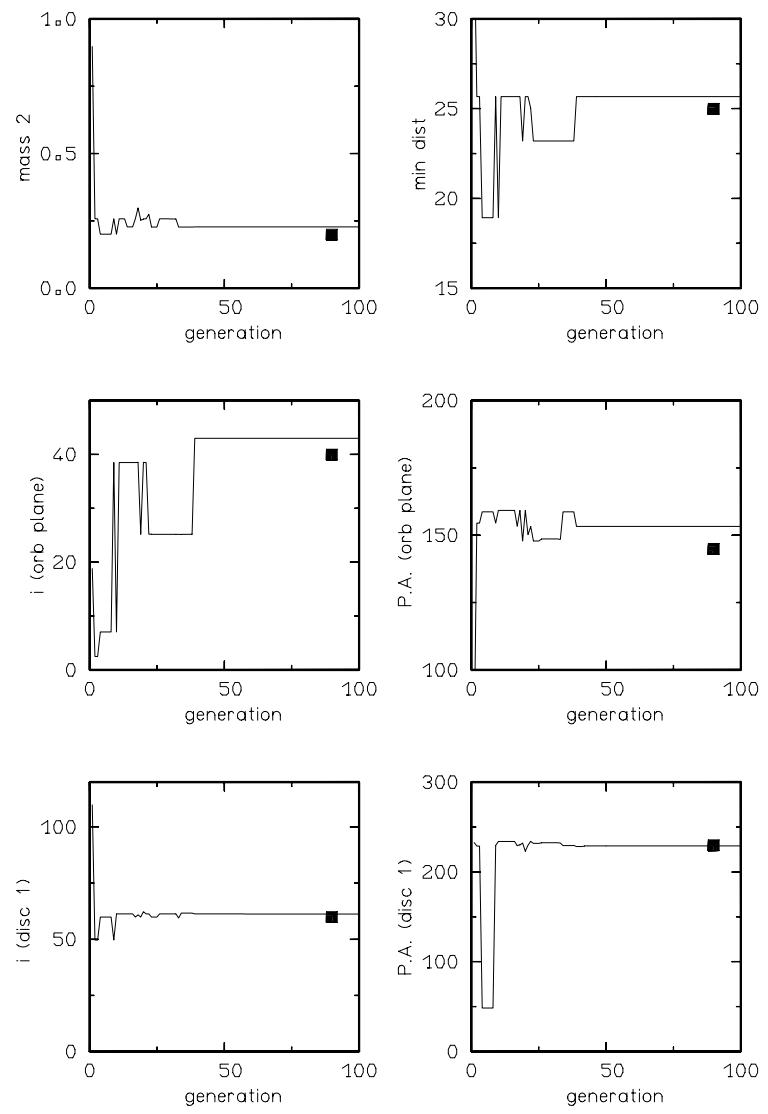

Fig. 11. Development of six parameters of the best fit model during a GA run. The parameters are the mass of the secondary galaxy (upper left), the minimum distance (upper right), the inclination (middle left) and position angle (middle right) of the orbital plane, and the inclination (lower left) and position angle (lower right) of the disc of the primary galaxy. The filled squares show the parameters of the fitted artificial reference model

model $^{3}$ - displayed in the upper left diagram - as well as the GA models were calculated with 900 test particles. This makes the streamers less prominent, but still discernible. The next plots show the GA status at different generations: at generation 1 , the best model is created out of the randomly chosen initial parameter sets. However, already by the second generation, i.e. after the first application of the reproduction operator, the best model clearly exhibits the north-eastern streamer. At generation 11 , even the weaker vertical feature and the bridge feature appear. At the end of this GA run the particle distribution of the original model $\mathrm{A}$ and the best fit of the GA are almost identical, at least compared by eye. The fitness of the best fit has strongly increased from 0.04 to 0.14 where values above 0.1 indicate a very good fit. Most of the improvements were found during the first 50 generations, i.e. analyzing 5000 models. After that generation, the homogeneity of the population led to inbreeding which

\footnotetext{
${ }^{3}$ We were only able to perform a uniqueness check of our preferred scenario using the best model as reference. A better model might be determined using the original VLA data as reference.
} 

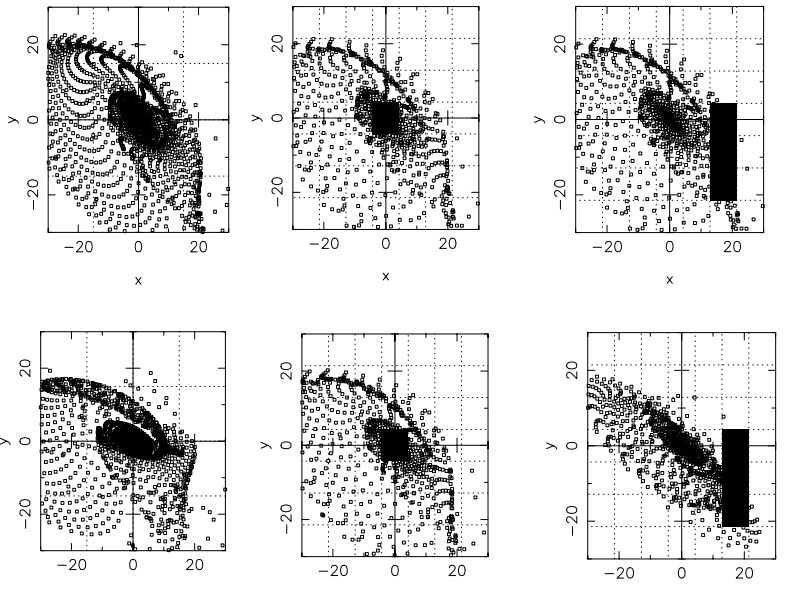

Fig. 12. Test of the capability of the GA (lower row) to recover original data when these data (upper row) are of low quality or incomplete. Shown are runs with low resolution, i.e. a discretization on a $4 \times 4$ grid (left column), a bad pixel (here an omitted central pixel, middle column), and a missing structure (the vertical streamer, right column)

strongly prohibits the appearance and spread of new (better) parameters. However, this is not a problem for the models here, because a very good fit to all parameters was already found after 40 generations (Fig. 11). The relative deviation of the derived parameters from the original values is less than $15 \%$ in all cases, and for many is better than $5 \%$.

In a series of different GA runs, we varied the population size, the number of generations or particles, the parameter combination encoded on chromosomes, the fitness function, and the random initial population. In almost all of them the best fit gave an acceptable fit of the reference model which demonstrates the capability of GAs to recover the parameters, even for weak encounters. The models which failed suffered from inbreeding or from a fitness function which did not discriminate sufficiently between high and low quality fits. Moreover, a different region in parameter space producing a good fit $(f>0.1)$ was never found. Thus, the model A - or more exact the corresponding intensity map - seems to result from a unique parameter set. However, this is only a motivated guess, not a mathematical proof!

Another interesting question is how strongly do the GA results depend on the completeness or accuracy of the data. We studied this in two ways: first, we used a coarser grid $(4 \times 4)$ in order to mimic a lower resolution of observational data (left column in Fig. 12). The GA is then only qualitatively able to reproduce the reference model. Obviously, too much information is lost when the resolution becomes too small. On the other hand, an increase of the number of cells to $10 \times 10$ did not improve the fit found on a $7 \times 7$ grid, but introduced more noise into the intensity maps. In the second test we discarded one or several grid cells from the fitness calculation (middle and right column in Fig. 12). Omitting one cell does not affect the final fit, independent of the location of the cell (here, the center). However, if a whole feature, such as the vertical streamer, is neglected, the GA is unable to recover the original data.

\subsection{Self-consistent models "sin gas"}

The self-consistent models in this paper were performed by a direct $N$-body integration using the GRAPE3af board in Kiel for the calculation of the gravitational forces and the potentials. The time integration was done by a leap-frog scheme with a fixed timestep of $1.78 \mathrm{Myr}$. The GRAPE board uses intrinsically a Plummer softening, the softening length was chosen to be $0.2 \mathrm{kpc}$. These choices give a total energy conservation of better than $1 \%$ for the whole simulation.

Different to the restricted $N$-body models, the set-up of the initial particle configuration for self-gravitating systems is far from being trivial. In principle, any stable stationary stellar system fulfills the collisionless Boltzmann equation. Thus, a solution of the latter could be used as a trial galactic system. From observations of the Milky Way it is known, however, that even if the Galaxy is axisymmetric, three integrals of motion are required to construct a stationary model (e.g. Binney \& Tremaine 1987). Unfortunately, only two are known analytically (the energy and the $z$-component of the angular momentum). Additionally, even if a particle configuration is generated from a distribution function depending on the integrals of motion, it is unclear whether it is stable against perturbations which might lead to e.g. the Toomre instability or the bar instability. Thus, on the timescale of interest one has to check the stability by numerical simulations.

In this paper we use the models of Kuijken \& Dubinski (1995) consisting of three components which are determined self-consistently from the Boltzmann equation: the bulge follows a King model and the halo a lowered Evans model. For the disc the third integral is approximated by the $z$-energy, i.e. the energy in vertical oscillations. The advantage of Kuijken \& Dubinski's method is the possibility to specify parameters like scale length or scale height of the disc directly, whereas many other methods (e.g. Barnes 1988) not starting in exact virial equilibrium readjust the mass distribution on a dynamical timescale, which means less control on the structural parameters.

Our initial model of NGC 4449 is derived from the observational data of Bajaja et al. (1994) and Hunter et al. (1998). The total mass is set according to the dynamical mass of $710^{10} M_{\odot}$ within $32 \mathrm{kpc} .90 \%$ of this mass is attributed to the dark halo, $3 \%$ to the disc and $7 \%$ to the bulge component. The radial extent of the halo was set to $32 \mathrm{kpc}$. The disc modelled here should not be confused with the optical disc of the galaxy. Since we are interested in the tidal response of the outer regions, especially the formation of the streamers, we did not model the inner (optical) region of NGC 4449, i.e. the stellar disc. The missing signatures of any tidal features in the optical 
image demonstrate that the stellar body of NGC 4449 is not affected by the interaction. For the same reason we did not consider the counter-rotation of the inner region. However, its gravity is taken into account by means of the bulge and halo contributions to the overall rotation curve. The radial extension of the bulge is $3 \mathrm{kpc}$. The scale length of the extended (HI) disc was varied between 10 and $30 \mathrm{kpc}$, the outer edge was set to $40 \mathrm{kpc}$.

The long-term stability was checked by simulations of an isolated disc with $N=18000$ particles (8000 for the disc, 4000 for the bulge and 6000 for the halo). Within 1.5 Gyr the Lagrange radii at $10 \%$ and $90 \%$ mass vary only by $2-4 \%$. They show no systematic trend except for a small expansion during the first $100 \mathrm{Myr}$ which is probably caused by gravitational softening. Due to two-body relaxation the scale height of the disc increases by almost a factor of 2 within 1.5 Gyr. However, this heating is not expected to alter the result of the dominant interaction significantly. The Fourier amplitude $C_{2}$, defined by $C_{m} \equiv\left(\int_{\text {disc }} \Sigma(R, \phi) r \mathrm{~d} r \mathrm{e}^{-i m \phi} \mathrm{d} \phi\right) /\left(\int_{\text {disc }} \Sigma(R, \phi) r \mathrm{~d} r \mathrm{~d} \phi\right)$ (with the surface density $\Sigma$ ), is always less than $5 \%$, in agreement with no significant bar or spiral pattern built up during the simulation.

Figure 13 shows a comparison of restricted $N$-body simulations with self-consistent models. Except for the more diffuse structure in the latter, the large-scale features are very similar in both simulations. The streamer structure can be found in both self-consistent simulations, as the surface plots demonstrate. The fact that the streamers are better resolved in the simulation with an eccentricity $\epsilon=0.5$, is partly due to the increased number of particles $(80000)$ used for this simulation. The simulations for the parabolic orbits were performed with 8000 (disk) particles for both, the restricted and the self-consistent simulations. The latter clearly demonstrates the ability of the restricted $N$-body models to resolve structures, even when a small number of particles is used. It is remarkable that the extended dark halo which has been applied for the selfconsistent models does not strongly affect the results as the comparison of the $\epsilon=0.5$-models shows: the vertical and the north-eastern streamer, the mass ejected to north of the north-eastern streamer, the small bridge between the central region and the eastern tip of the streamer, the extent of the $\mathrm{HI}$ distribution south and south-east to the center show up in both simulations at almost the same locations. The only significant difference is that the north-eastern streamer is less straight in the self-consistent model than in the restricted $N$-body simulation. This very good agreement confirms the applicability of the restricted $N$-body method here. Another interesting aspect concerns the response of the halo to the intruder. Unlike to the disc, the halo remains almost unchanged by the encounter (Fig. 14). This behaviour allows the application of a rigid galactic potential which has been used in the restricted $N$-body simulations.
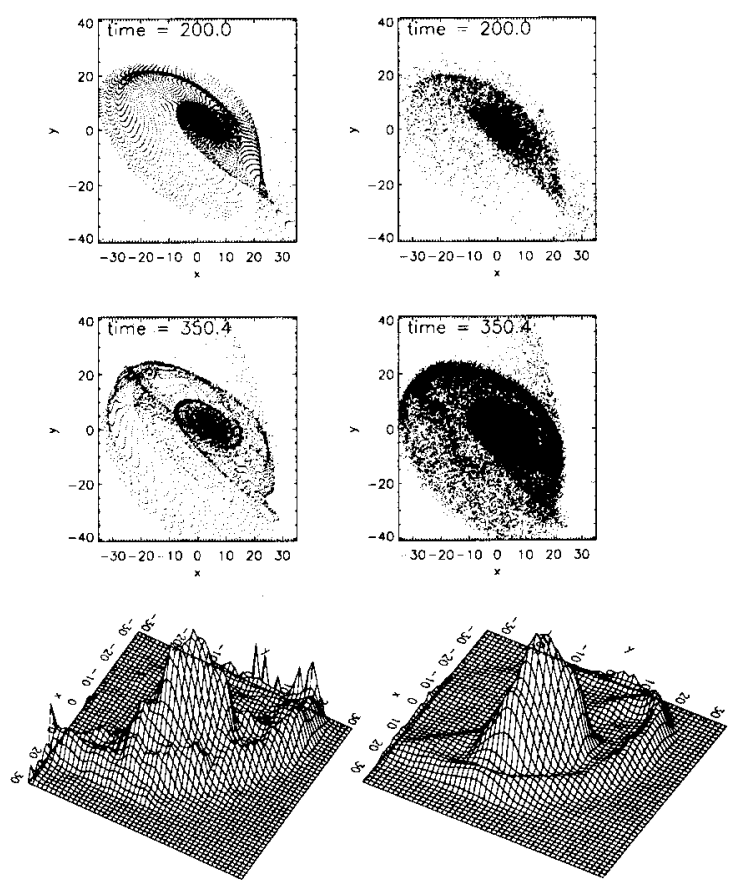

Fig. 13. Comparison of restricted $N$-body models (left) with self-consistent simulations (right) for a parabolic encounter (upper row) and an eccentricity of $\epsilon=0.5$ (middle row). The projected distance to the perturber (here about $40 \mathrm{kpc}$ ) defines the time of the snapshot. The lower row displays the normalized intensity profiles for the self-consistent simulations (left: $\epsilon=1$, right: $\epsilon=0.5$ ). Note that the surface plots are seen from north-west in order to emphasize the coherent streamer structure

\subsection{Self-consistent models "con gas"}

For the gasdynamical models we use a sticky particle scheme which treats each particle of the HI disc as a gaseous cloud. The radius of these clouds is assumed to follow $R_{\mathrm{cl}}=20 \cdot \sqrt{M_{\mathrm{cl}} / 10^{6} M_{\odot}} \mathrm{pc}$ which is derived from the mass-radius relation of Rivolo \& Solomon (1987) corrected for glancing collisions $\left(M_{\mathrm{cl}}\right.$ is the mass of a gas cloud). When the distance of two clouds falls below the sum of their radii, merging of the clouds is allowed, if their relative orbital angular momentum is smaller than the maximum angular momentum a merged cloud can acquire before break-up. If this angular momentum criterion prevents a merger, the clouds keep their kinematical data, i.e. no energy is dissipated. Star formation is only qualitatively included by adopting an upper mass limit of $710^{8} M_{\odot}$ for clouds. If they exceed this limit no further merging is allowed, i.e. they are treated as stars. However, the star formation criterion is not important for the models of this paper. (For details of the cloud model or the merging mechanism see Theis \& Hensler 1993). This dissipative scheme has been successfully applied to collapsing systems reproducing many observed properties of spiral and elliptical galaxies. For the long-term evolution of barred disc galaxies, a comparison of this scheme with that of Palouš et al. (1993) for their standard coefficients 

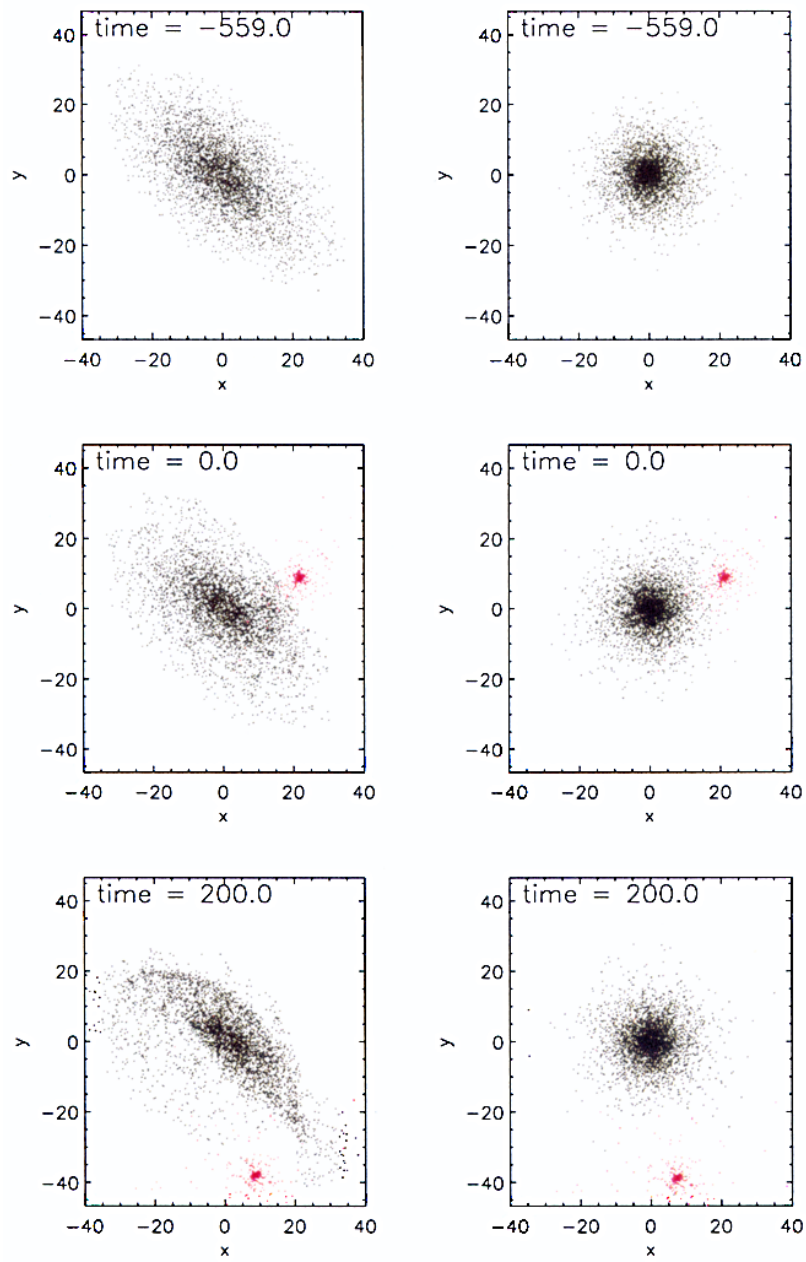

Fig. 14. Self-consistent $N$-body simulation "sin gas": projection of the particle positions onto the plane-of-sky at different times: initially (upper row), at closest approach (middle row) and at the projected distance of $41 \mathrm{kpc}$ (lower row). The left column shows the disc particles, whereas the right column displays the halo particles

of restitution of $\beta_{\mathrm{r}}=\beta_{\mathrm{t}}=0.2$ gives basically the same results (Jungwiert, private communication). Thus, the chosen model should be applicable for a reliable determination of the difference between gas dynamical and purely stellar dynamical models.

A comparison of gaseous models with dissipationless models shows no significant difference (Fig. 15). Due to the low gas densities only about $20-30 \%$ of the clouds undergo inelastic collisions which mainly occur in the denser central regions. Thus, the outer HI halo is only very weakly influenced by (sticky particle) gas dynamics and the application of purely stellar simulations for the $\mathrm{HI}$ is possible.

\section{Discussion}

\subsection{Interaction between NGC 4449 and DDO 125?}

The previous simulations demonstrate that models based on an encounter scenario are able to reproduce the morphology of the three streamers. Furthermore, the
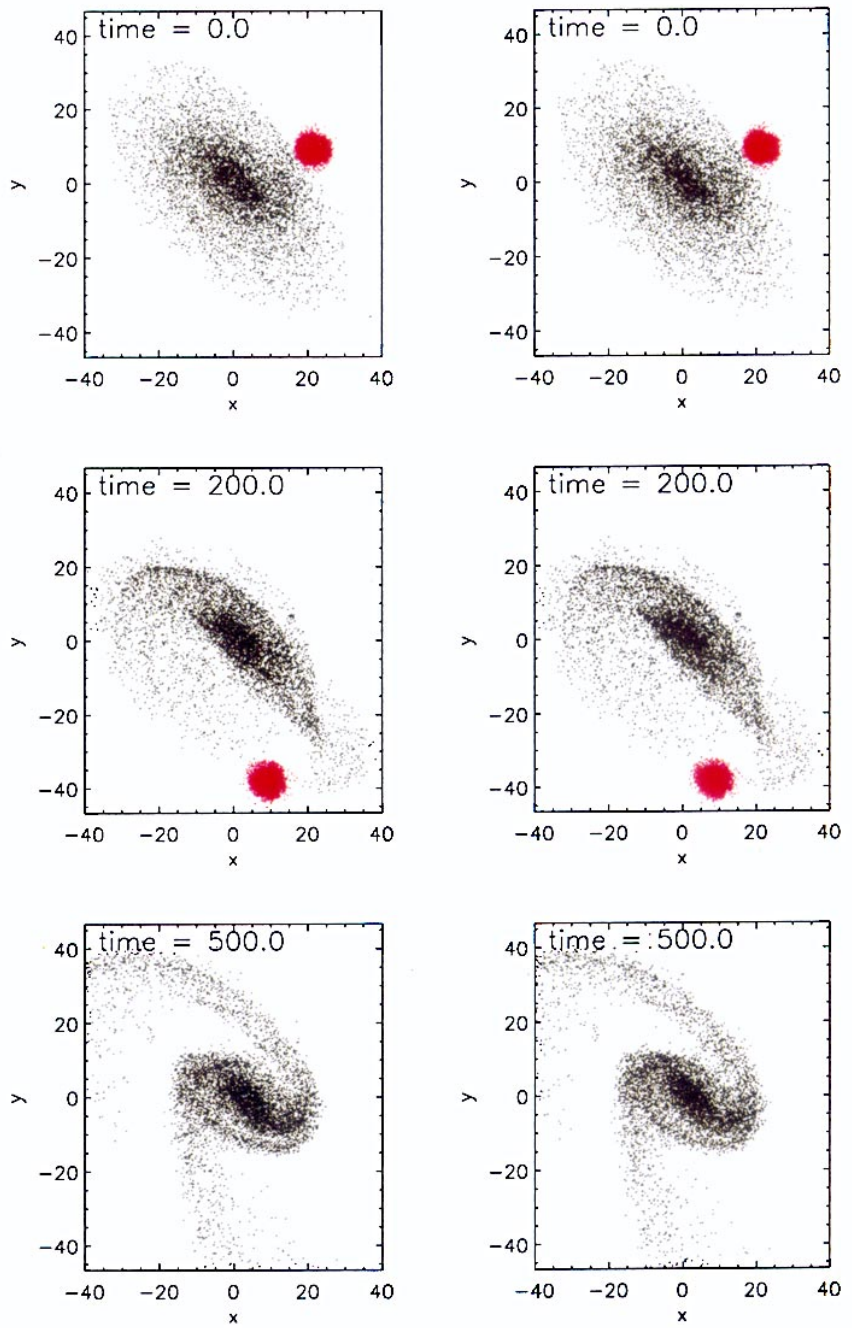

Fig. 15. Projection of the particle positions onto the plane-ofsky at different times: at closest approach (upper row), at the projected distance of $41 \mathrm{kpc}$ (middle row) and at the end of the simulation (lower row). Both models include self-gravity. The left column shows a purely stellar simulation, whereas the right column includes gas dynamics by means of sticky particles

interaction puts gas to the south-west of NGC 4449, where a clumpy HI distribution is observed (Hunter et al. 1998). Though its detailed patchy structure has not been recovered by the models, an initial gas distribution less regular than the smooth disc-like one used for the numerical models might be responsible for this deviation.

The parameter study showed that small variations in the parameters give HI distributions which are not in agreement with the observations. Especially, the strong dependence of the final structure on the initial orientation of the HI disc confines its inclination and position angle within $10^{\circ}$ to the values derived independently from the observations. This remarkable coincidence would occur necessarily in the encounter scenario, whereas in the infall scenario it happens just by chance. A second critical feature is the abrupt direction change of the streamers. In the infall scenario they mark the path of the perturbed 
gas which collapses to the galactic center. However, it is hard to imagine how a realistic static potential could create orbits with such strong direction changes as e.g. the one from the vertical to the bridge feature. Moreover, due to the large distance of these features from the galactic center the potential should be more or less spheroidal or already spherical, which makes it even more difficult to produce such orbits.

The most critical parameter for the interaction scenario is the mass ratio $q$ of DDO 125 and NGC 4449. The simulations demonstrate that a mass ratio smaller than $10 \%$ cannot reproduce the streamer structure. In order to determine $q$, both galactic masses have to be known. In Bajaja et al.'s (1994) estimate for the mass of NGC 4449 they assumed that the $\mathrm{HI}$ is in rotational equilibrium even at a distance of $32 \mathrm{kpc}$. However, in the case of an interaction with DDO 125 the velocities at large distances are strongly affected by the interaction and they are definitely not in rotational equilibrium (as the ejection of the tidal arms in a continuation of the simulations demonstrates). Hence, the derived mass of $710^{10} M_{\odot}$ is at best an upper mass limit of NGC 4449. A better guess is the dynamical mass inside the central gaseous ellipse which seems to be unaffected by interaction. E.g. the dynamical mass inside $11 \mathrm{kpc}$ would be a factor 3 smaller than Bajaja's value.

Even more difficult is the mass determination of DDO 125. Observations by Tully et al. (1978) gave a dynamical mass of $510^{8} M_{\odot}$ inside the optical radius of DDO 125. However, Ebneter et al. (1987) reported a linear increase in the rotation curve exceeding the range observed by Tully et al. by a factor of two. Moreover, they found no hint of a flattening or turn-over in DDO 125's rotation curve. This gives a lower mass limit of $410^{9} \mathrm{M}_{\odot}$. Combining the mass estimates yields a lower limit of the mass ratio of $6 \%$ when using Bajaja's mass determination of NGC 4449. A more realistic mass ratio might be derived from the ranges of rigid rotation resulting in $q \approx 18 \%$. Thus, observationally it cannot be excluded that the mass ratio is below the critical one for the interaction scenario, however, the more realistic estimates seem to be in agreement with the values found in the simulations. The situation could be clarified when a reliable mass determination of DDO 125 is available.

\subsection{Origin of the extended HI-distribution around NGC 4449}

Another important question is related to the initial conditions of the previous simulations, i.e. the origin of the extended HI distribution. In principle, there are several possibilities:

Idea 1. The HI comes from DDO 125. In a previous encounter $(\epsilon<1)$ the gas was stripped off from DDO 125 . Due to the long orbital period of e.g. 3.6 Gyr for $\epsilon=0.5$ the gas has enough time to settle down in a regular disclike structure. However, the amount of HI gas in DDO 125 is much smaller than the gas seen around NGC 4449.
It seems unlikely that DDO 125 could have survived the loss of such a large fraction of its gas content without disruption.

Idea 2. The HI comes from NGC 4449. Though a star forming dwarf galaxy drives a galactic wind e.g. by means of type II supernovae, a detailed fine-tuning of the energy injection would be required to prevent a blow-out of the HI gas, especially if it acquired enough energy to form such an extended system. Moreover, the counter-rotation of the HI outside and inside the optical radius of NGC 4449 makes this scenario not very appealing.

Idea 3. The HI gas stems from a previous minor merger with NGC 4449. Though a satellite galaxy can be disrupted by the tidal field of NGC 4449, it is very unlikely that it is already disrupted far outside the galactic center where the HI gas is detected.

Idea 4. The HI originates from the collapse of a dwarf companion. A dwarf galaxy forms in the vicinity of NGC 4449 similar to the formation scenario of compact ellipticals suggested by Burkert (1994). By this it undergoes a strong collapse which leads to mass ejection due to violent relaxation. In principle, a mass loss of up to $40 \%$ of the companion could occur, providing enough gas to explain the HI. However, where is the dwarf today? Excluding an unlikely radial orbit, the dwarf's orbit could in principle decay by dynamical friction. However, due to the large distance to NGC 4449 the decay time should be at least several orbital periods, which exceeds the Hubble time.

Idea 5. Another major encounter. Although NGC 4449 is a member of the loose Canes Venatici group, there seems to be no close galaxy which has a radial velocity in agreement with the rotational sense of the gaseous disc in NGC 4449. The only candidate is the ringed Sab-type galaxy NGC 4736, located about 5.5 degrees south-east of NGC 4449. The projected distance of $350 \mathrm{kpc}$ and the differential radial velocity of $\Delta v=101 \mathrm{~km} \mathrm{~s}^{-1}$ (NED) is compatible with a scenario in which both galaxies experienced a close encounter about $3.510^{9}$ years ago on a hyperbolic orbit (Kohle 1999). Placing NGC 4736 at a distance of $4.4 \mathrm{Mpc}$, its total $\mathrm{HI}$ mass is $4.210^{8} M_{\odot}$ (Bosma et al. 1977; Mulder \& van Driel 1993), which is quite low for Sab-type galaxies (Solanes et al. 1996). Might it be that NGC 4449 formed its halo out of gas stripped from another galaxy?

\section{Summary and conclusions}

Several $N$-body methods are combined in order to develop a method for the determination of the parameters of interacting galaxies. This method is applied to the HI distribution of NGC 4449. In a first step, the fast restricted $N$-body method is used to confine a region in parameter space which reproduces the main observed features. In a second step, a genetic algorithm (also using restricted $N$ body calculations) is employed which allows, in principle for both, an automatic fit of observational data even in a high-dimensional parameter space and/or a uniqueness 
test of a favoured parameter combination (only the latter has been done in this paper). For a genetic algorithm one typically has to follow a population of (at least) 100 members for 100 generations in order to get a good fit, provided the data are sufficiently accurate. Missing single pixels do not inhibit the parameter determination, as long as the key features are included in the data. Since a typical restricted $N$-body simulation takes a few CPU-seconds on a Sparc workstation, the whole fitting procedure is finished after 3-6 CPU-hours.

In the third step, the results of the previous steps are compared with detailed self-consistent $N$-body simulations. In the case of NGC 4449, they show that the restricted $N$-body calculations are reliable models for this encounter. The comparison with the sticky particle models demonstrates that the HI gas can be modeled without any restriction by a purely stellar dynamical approach, provided the encounter is weak, as in the case of NGC 4449 and DDO 125.

From the previous simulations, we conclude that the extended HI features observed in NGC 4449 are created by an encounter with DDO 125. Prior to the encounter, the HI gas formed an extended, almost homogenous disc with a large scale length of about $30 \mathrm{kpc}$ and a radial extension of $40( \pm 10) \mathrm{kpc}$. The orientation of the disc is in agreement with the orientation of the inner ellipsoidal HI distribution. The orbital plane has an inclination angle of about $40^{\circ}\left( \pm 10^{\circ}\right)$. The eccentricity is in the vicinity of a parabolic encounter, but favouring the region of elliptical orbits $(0.5<\epsilon<1.5)$. The apocenter distance of the galaxies is about $25( \pm 5) \mathrm{kpc}$. The closest approach happened 3.5-6.2 $10^{8}$ yr ago (depending on eccentricity). The mass ratio of both galaxies must be approximately 0.2 (and cannot be smaller than 0.1).

Acknowledgements. The simulations were partly performed with the GRAPE3af special purpose computer in Kiel (DFG Sp345/5). The authors are grateful to Deidre Hunter, Jay Gallagher, Uli Klein and Hugo van Woerden for stimulating discussions about NGC 4449. We are also greatly indebted to Konrad Kuijken and John Dubinski who made their program for the generation of initial particle distributions available to the public, and Paul Charbonneau and Barry Knapp for providing their PIKAIA code. S.K. acknowledges DFG grant III GK-GRK 118/2. This research has made use of the NASA/IPAC Extragalactic Database (NED) which is operated by the Jet Propulsion Laboratory, California Institute of Technology, under contract with the National Aeronautics and Space Administration. Finally, we thank the language editor of A\&A for a careful reading (and improving) of the manuscript.

\section{Appendix A: The genetic algorithm}

In the following sections we briefly describe the main ingredients of our genetic algorithm. The GA used here is a slightly modified version of P. Charbonneau's code PIKAIA (for details see Charbonneau 1995). The interface between the GA and the simulations, i.e. the fitness determinations, is performed in a similar way as in Wahde (1998), however the coding of genes as well as the choice of parameters used as genes differ from Wahde's approach.

\section{A.1. Fitness and parent selection}

In order to determine the fitness of an individual (i.e. a special parameter set), the $N$-body simulation has to be performed and compared with the observation. Generally, this is done by mapping the particles on a Cartesian grid. However, here a quadratic grid of size $60 \mathrm{kpc}$ centered on NGC 4449 is used. The resolution of the grid was set to $7 \times 7$ in order to allow for a better resolution of the tidal features of an encounter. For each grid cell the intensity was calculated by summing the masses of the individual particles in that cell. The conversion to observed intensities can be performed by assuming a mass-to-light ratio for the particles (or treating it as another free parameter) or by normalizing the intensities. Here the intensities are normalized to the total intensity of the whole grid. The quality can be measured by the relative deviation $\delta$ of the intensities in both maps:

$\delta \equiv \sum_{i(\mathrm{cells})} \frac{\left|I_{\mathrm{ref}, i}-I_{\mathrm{mod}, i}\right|}{\max \left(I_{\mathrm{ref}, i}, I_{\mathrm{mod}, i}\right)}$.

The fitness $f$ is then estimated by

$f \equiv \frac{1}{1+\delta}$

In the case of an impossible parameter configuration (e.g. a circular orbit with a distance less than the observed projected distance) or any convergence problems the fitness of that point in parameter space is set to zero, prohibiting any offspring. Typically, only a few models in $10^{4}$ parameter sets fail, most of them in the first few generations before the system starts to approach the parameter space region of interest.

In order to determine the parents of the next generation, the members of the actual generation are ranked by their fitness. The parents are then selected randomly, whereas the probability of being chosen is proportional to the rank (roulette-wheel selection).

A typical example of the status of the GA after a few generations is shown in Fig. A.1. In the left column the positions of the original data are discretized on the grid, yielding the intensities displayed on the lower left. Though the resolution of the grid (about $8.6 \mathrm{kpc}$ ) exceeds the thickness of the tidal structures, the large scale pattern is quickly found: in the middle column the best fit of the actual (here 7th) generation already agrees qualitatively with the original. This demonstrates that large scale features already present in intermediate resolution maps can be sufficient to constrain the dynamics. This is in agreement with Wahde's (1998) calculations for equal mass galaxies: he recovered orbital parameters by using a $4 \times 4$ grid. The quality of our fits is calculated only by a comparison of the lower two intensity diagrams. The fitness distribution in the population resembles a Gaussian at the beginning of the fitting procedure. 

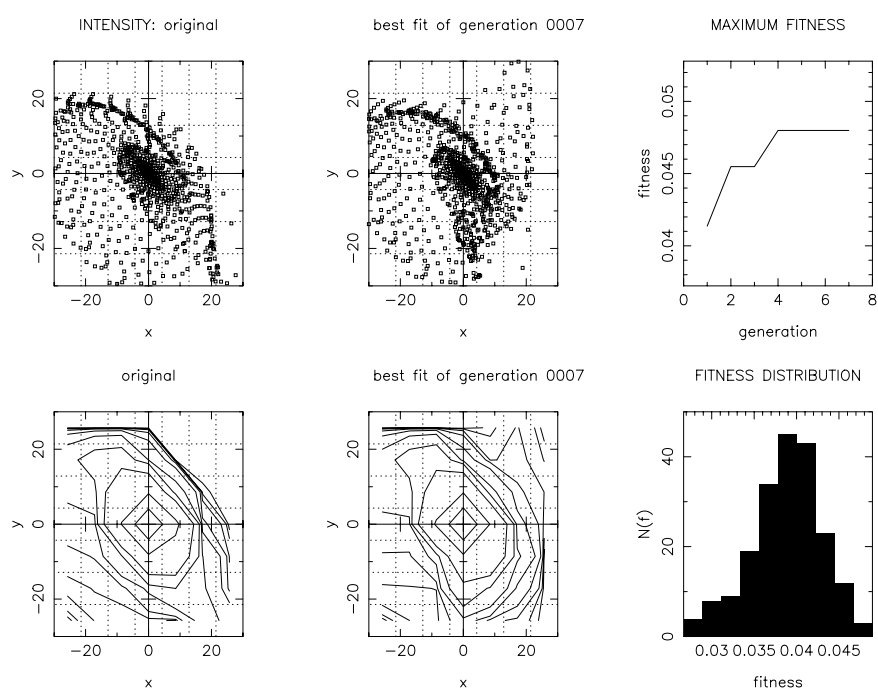

Fig. A.1. Fitness and best fit of an early generation during the GA fitting procedure. The figure displays the artificial original data (left column: positions of the particles in the $x$-y-plane (upper left) and the resulting intensity contour lines (linear spacing, lower left), the best fit of the actual generation (middle column) and information about the fitness history, i.e. the maximum fitness (upper right) and the actual fitness distribution (lower right). The underlying grid in the left and middle column is used to determine the intensities

\section{A.2. Coding of parameters}

In organic systems the information is coded in genes in the form of sequences of the four nucleotide bases where a triplet of them (a codon) encodes one amino acid, the building block of proteins. The genes themselves are arranged in one or several chromosomes which contain all the genetic information (or the genotype). The phenotype which is subject to the selection process is the result of the genetic information and the interaction with its environment.

Translated to our fitting problem, the phenotype is the final intensity map created by a special choice of parameters or initial conditions. The latter correspond to the proteins which physically express the genetic information. The mapping between the parameters and the genes or the number of chromosomes is not fixed in GAs. Many GAs apply a binary alphabet to encode the parameters (e.g. Holland 1975; Goldberg 1989). In Charbonneau's program PIKAIA a decimal encoding is implemented. For our calculations we combined four decimal digits giving a number between zero and one. This gene has been mapped to a real parameter by a linear or logarithmic transformation, e.g. the orbital inclination is mapped linearily to the range $\left[0^{\circ}\right.$, $180^{\circ}$ ] (Fig. A.2). In general, the linear mapping of parameter $x$, which is allowed to vary between $x_{\text {low }}$ and $x_{\text {high }}$, is performed by

$x=x_{\text {low }}+g \cdot\left(x_{\text {high }}-x_{\text {low }}\right)$.

Here $g$ denotes the value of the gene which is in the range $[0,1]$. Similarly, the logarithmic mapping is done by



Fig. A.2. Schematic diagram for the coding of parameters on a numerical chromosome
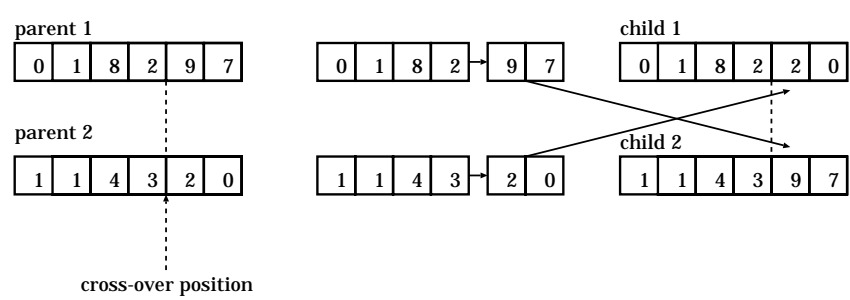

Fig. A.3. Schematic diagram for the cross-over operation

replacing $x$ by $\log (x)$. All the genes are assumed to be located in a linear fashion on a single chromosome. Unlike organic evolution, the phenotype is completely determined by the genotype, no environmental influence on the individual acts after its genotype is fixed.

\section{A.3. Cross-over}

The main difference between evolutionary strategies and genetic algorithms is the sexual reproduction in the framework of GAs. This means that the chromosomes of the parents are combined in a new manner by means of a cross-over mechanism in addition to mutation. The principle idea is illustrated in Fig. A.3: after determining the two parents a random position on the chromosomes is selected. At this position the chromosomes are split into two fragments which are exchanged among the chromosomes resulting in two new ones.

\section{A.4. Mutation}

In order to introduce completely new information into a genepool a mutation process, i.e. a random change of the information on the chromosomes is introduced (Fig. A.4). For each position on a chromosome, mutation is applied with a small probability (typ. $p_{\text {mut }} \approx 0.5 \%$ ). After selecting a position for mutation, an integer random number in the range $[0,9]$ replaces the original value at that position. Thus, a real change in the genetic information takes place with a probability of $90 \%$, even if that position of a gene is subject to mutation. The overall probability for at least one real mutation on a chromosome coding 6 parameters by 4 digits is about $11 \%$. Thus, cross-over is the most important process introducing diversity into the population, whereas mutation affects only each 9th member of 
the population. In principle, the mutation rate can be increased, but the price would be a more or less random search in parameter space which is already after a few generations inferior to the oriented search of a GA method (Wahde 1998).

A principal danger of GAs is inbreeding, i.e. the homogenization of the genepool by one or a few dominant genes. If they successfully replace other genes, cross-over does not introduce sufficient (or - if inbreeding is complete - any) diversity into the population and thus any further improvement of the chromosomes is prohibited. The only way to overcome this situation is to introduce more diversity by mutation. Therefore, PIKAIA allows for a variable mutation rate which increases the mutation probability $p_{\text {mut }}$ whenever the fitness distribution becomes narrow, i.e. inbreeding is suspected to operate. On the other hand, $p_{\text {mut }}$ is decreased if the fitness distribution becomes very broad, i.e. the randomization is dominant.

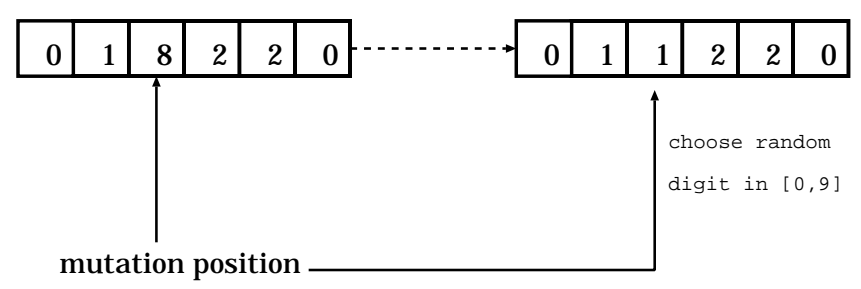

Fig. A.4. Schematic diagram for the mutation process

\section{A.5. Determination of the next generation}

After $N_{\text {pop }}$ children have been created, the old generation is completely replaced by the next generation (full generational replacement). The only exception is the survival of the fittest member of the parent generation, if it is fitter than the child which would replace it (elitism). This mechanism prevents the system from forgetting successful solutions.

The initial population is randomly chosen in the accessible parameter space. A standard population size is about $N_{\text {pop }}=100-200$ members. The GA converges typically after about $N_{\text {gen }}=100$ generations. Thus, $10^{4}$ or a few $10^{4} \mathrm{~N}$-body simulations are required for a single GA fit. For a genetic algorithm simulation with 900 particles (and no offset for an inner edge) 2 CPU-seconds are required per simulation compared to $3 \mathrm{CPU}$-hours on a GRAPE3. Thus, self-consistent $N$-body models, such as the mentioned GRAPE models, would still need 3.4 years, whereas the restricted $N$-body models reduce the CPUrequirement to 5.6 hours on a $150 \mathrm{MHz}$-Sparc10.

\section{References}

Arp, H. 1966, Atlas of peculiar galaxies, Pasadena, Caltech (also Arp, H. 1966, ApJS, 14, 1)

Bäck, Th. 1996, Evolutionary Algorithms in Theory and Practice (Oxford University Press, Oxford)
Bajaja, E., Huchtmeier, W. K., \& Klein, U. 1994, A\&A, 285, 388

Barnes, J. 1988, ApJ, 331, 699

Barnes, J., \& Hernquist, L. 1996, ApJ, 471, 115

Barnes, J., \& Hut, P. 1986, Nature, 324, 446

Binney, J., \& Tremaine, S. 1987, Galactic Dynamics (Princeton University Press, Princeton)

Bomans, D., \& Chu, Y.-H. 1997, AJ, 113, 1678

Bosma, A., van der Hulst, J. M., \& Sullivan, W. T. 1977, A\&A, 57,373

Burkert, A. 1994, MNRAS, 266, 877

Casoli, F., \& Combes, F. 1982, A\&A, 110, 287

della Ceca, R., Griffiths, R. E., \& Heckman, T. M. 1997, ApJ, 485,581

Charbonneau, P. 1995, ApJS, 101, 309

Crillon, R., \& Monnet, G. 1969, A\&A, 1, 449

Ebneter, K., Davis, M., Jeske, N., \& Stevens, M. 1987, BAAS, 19, 681

Fisher, J. R., \& Tully, R. B. 1981, ApJS, 47, 139

Goldberg, D. E. 1989, Genetic Algorithms in Search, Optimization, \& Machine Learning (Addison-Wesley, Reading)

Hernquist, L. 1987, ApJS, 64, 715

Hernquist, L. 1990, J. Comp. Phys., 87, 359

Hernquist, L., \& Katz, N. 1989, ApJS, 70, 419

Hernquist, L., \& Ostriker, J. P. 1992, ApJ, 386, 375

Hill, R. S., Home, A. T., Smith, A. M., et al. 1994, ApJ, 430, 568

Holland, J. 1975, Adaptation in natural and artificial systems (University of Michigan Press, Ann Arbor)

Holmberg, E. 1941, ApJ, 94, 385

Hunter, D. A., \& Gallagher, J. S. 1997, ApJ, 475, 65

Hunter, D. A., Gillet, F. C., Gallagher, J. S., Rice, W. L., \& Low, F. J. 1986, ApJ, 303, 171

Hunter, D. A., \& Thronson, H. A. 1996, ApJ, 461, 202

Hunter, D. A., Wilcots, E. M., van Woerden, H., Gallagher, J. S., \& Kohle, S. 1998, ApJL, 495, L47

Kant, I. 1755, in Allgemeine Naturgeschichte und Theorie des Himmels oder Versuch von der Verfassung und dem mechanischen Ursprunge des ganzen Weltgebäudes nach Newtonischen Grundsätzen abgehandelt, ed. H. Ebert, 1890 (Verlag Wilhelm Engelmann, Leipzig)

Karachentsev, I. D., \& Drozdovsky, I. O. 1998, A\&AS, 131, 1

Klein, U., Hummel, E., Bomans, D., \& Hopp, U. 1996, A\&A, 313,396

Kohle, S., Klein, U., Henkel, C., \& Hunter, D. A. 1998, in Proc. of The Magellanic Clouds and Other Dwarf Galaxies, ed. T. Richtler, \& J. M. Braun (Bad Honnef), 265

Kohle, S., Ph.D. Thesis, University of Bonn, 1999

Kuijken, K., \& Dubinski, J. 1995, MNRAS, 277, 1341

Mulder, P., \& van Driel, W. 1993, A\&A, 272, 63

Noguchi, M. 1988, A\&A, 203, 259

Palouš, J., Jungwiert, B., \& Kopecký, J. 1993, A\&A, 274, 189

Pfleiderer, J., \& Siedentopf, H. 1961, Zs. f. Ap., 51, 201

Press, W. H., Teukolsky, S. A., Vetterling, W. T., \& Flannery, B. P. 1992, Numerical Recipes in FORTRAN - The Art of Scientific Computing (Cambridge University Press, Cambridge)

Rechenberg, I. 1965, Cybernetic solution path of an experimental problem, Royal Aircraft Establishment, Library Translation No. 1122, Farnborough

Rivolo, A. V., \& Solomon, P. M. 1987, in Molecular Clouds in the Milky Way and External Galaxies, ed. R. L. Dickman, et al. (Springer), 42 
Sasaki, M., Ohta, K., \& Saito, M. 1990, PASJ, 42, 361

Schwefel, H.-P. 1977, Numerische Optimierung von ComputerModellen mittels der Evolutionsstrategie, Birkhäuser, Basel

Sellwood, J. A. 1980, A\&A, 89, 296

Silk, J., Wyse, R. F. G., \& Shields, G. A. 1987, ApJ, 322, L59

Solanes, J. M., Giovanelli, R., Haynes, M. P. 1996, ApJ, 461, 609

Sugimoto, D., Chikada, Y., Makino, J., et al. 1990, Nature, 345,33

Theis, Ch., \& Hensler, G. 1993, A\&A, 280, 85

Theis, Ch., \& Kohle, S. 1998, in Proc. of The Magellanic Clouds and Other Dwarf Galaxies, ed. T. Richtler, \& J. M. Braun
(Bad Honnef), 209

Thomasson, M., \& Donner K. J. 1993, A\&A, 272, 153

Toomre, A., \& Toomre, J. 1972, ApJ, 178, 623

Tully, R. B., Bottinelli, L., Fisher, J. R., et al. 1978, A\&A, 63, 37

van Woerden, H., Bosma, A., \& Mebold, U. 1975, in La Dynamique des Galaxies Spirales, ed. L. Weliachew, 483

von Humboldt, A. 1850, in Kosmos, vol. III (Cotta'scher Verlag, Stuttgart), 178

Vorontsov-Velyaminov, B. A. 1959, Atlas and Catalog of Interacting Galaxies, Moscow, Sternberg Institute

Wahde, M. 1998, A\&AS, 132, 417 